Pacific

Journal of

Mathematics

DISCRETE PRODUCT SYSTEMS OF HILBERT BIMODULES

NEAL J. Fowler

Volume 204 No. 2

June 2002 


\title{
DISCRETE PRODUCT SYSTEMS OF HILBERT BIMODULES
}

\author{
Neal J. Fowler
}

\begin{abstract}
A Hilbert bimodule is a right Hilbert module $X$ over a $C^{*}$-algebra $A$ together with a left action of $A$ as adjointable operators on $X$. We consider families $X=\left\{X_{s}: s \in P\right\}$ of Hilbert bimodules, indexed by a semigroup $P$, which are endowed with a multiplication which implements isomorphisms $X_{s} \otimes_{A} X_{t} \rightarrow X_{s t}$; such a family is a called a product system. We define a generalized Cuntz-Pimsner algebra $\mathcal{O}_{X}$, and we show that every twisted crossed product of $A$ by $P$ can be realized as $\mathcal{O}_{X}$ for a suitable product system $X$. Assuming $P$ is quasi-lattice ordered in the sense of Nica, we analyze a certain Toeplitz extension $\mathcal{T}_{\mathrm{cv}}(X)$ of $\mathcal{O}_{X}$ by embedding it in a crossed product $B_{P} \rtimes_{\tau, X} P$ which has been "twisted" by $X$; our main Theorem is a characterization of the faithful representations of $B_{P} \rtimes_{\tau, X} P$.
\end{abstract}

\section{Introduction.}

Suppose $X$ is a right Hilbert module over a $C^{*}$-algebra $A$. If $X$ also carries a left action of $A$ as adjointable operators on $X_{A}$, we call $X$ a Hilbert bimodule over $A$. In [22], Pimsner associated with every such bimodule $X$ a $C^{*}$-algebra $\mathcal{O}_{X}$, which we shall call the Cuntz-Pimsner algebra of $X$, and showed that every crossed product by $\mathbb{Z}$ and every Cuntz-Krieger algebra can be realized as $\mathcal{O}_{X}$ for suitable $X$. He also commented that the algebras $\mathcal{O}_{X}$ include the crossed products by $\mathbb{N}$; that is, for each endomorphism $\alpha$ of a $C^{*}$-algebra $A$ there is a bimodule $X=X(\alpha)$ such that $\mathcal{O}_{X}$ is canonically isomorphic to the semigroup crossed product $A \rtimes_{\alpha} \mathbb{N}$ of $[\mathbf{6}, 24]$.

The work in this paper is motivated by the following observation, which also serves as our primary example. Suppose $\beta$ is an action of a discrete semigroup $P$ as endomorphisms of a $C^{*}$-algebra $A$. For each $s \in P$ let $X_{s}:=X\left(\beta_{s}\right)$ be the bimodule canonically associated with the endomorphism $\beta_{s}$. Then the family $X=\left\{X_{s}: s \in P\right\}$ admits an associative multiplication $(x, y) \in X_{s} \times X_{t} \mapsto x y \in X_{t s}$ which implements isomorphisms $X_{s} \otimes_{A} X_{t} \rightarrow X_{t s}$; we call a family with this structure a product system of Hilbert bimodules. (In this example $X$ is a product system over the opposite semigroup $P^{o}$.) Such families generalize the product systems 
of $[\mathbf{7}, \mathbf{8}, \mathbf{1 2}, \mathbf{1 0}]$, where the fibers $X_{s}$ are complex Hilbert spaces (bimodules over $\mathbb{C})$.

To each product system $X$ we associate a generalized Cuntz-Pimsner algebra $\mathcal{O}_{X}$. When $X$ is the product system associated with the semigroup dynamical system $(A, P, \beta), \mathcal{O}_{X}$ is canonically isomorphic to the semigroup crossed product $A \rtimes_{\beta} P$. Moreover, if we "twist" $X$ by a multiplier $\omega: P \times P \rightarrow \mathbb{T}$, then the corresponding Cuntz-Pimsner algebra is isomorphic to the twisted semigroup crossed product $A \rtimes_{\beta, \omega} P$. Our construction applies even when $A$ is nonunital provided each endomorphism $\beta_{s}$ extends to the multiplier algebra $M(A)$.

The aim of this paper is to take a first step towards analyzing the CuntzPimsner algebra of a product system $X$. Following Pimsner [22], we begin by studying the structure of its Toeplitz extension $\mathcal{T}_{X}$. This algebra is universal for Toeplitz representations of $X$; these are multiplicative maps whose restriction to each fiber $X_{s}$ is a Toeplitz representation in the sense of [13]. Our results generalize those of [12] for product systems of Hilbert spaces; indeed, much of the paper is devoted to adapting the methods of [12] to the bimodule setting. Thus our basic assumptions about the underlying semigroup $P$ are as in [12]: To allow our analysis to extend beyond the totally-ordered case, we assume that $P$ is the positive cone of a group $G$ such that $(G, P)$ is quasi-lattice ordered in the sense of Nica [20]. The class of such $(G, P)$ includes all direct sums and free products of totally ordered groups. We also impose a covariance condition, called Nica covariance, on Toeplitz representations of $X$. This means that the universal $C^{*}$-algebra $\mathcal{T}_{\text {cov }}(X)$ which we analyze is in general a quotient of $\mathcal{T}_{X}$. However, if $(G, P)$ is totally-ordered, then Nica-covariance is automatic, and hence $\mathcal{T}_{\text {cov }}(X)$ is the same as $\mathcal{T}_{X}$.

Our main goal is to characterize the faithful representations of $\mathcal{T}_{\text {cov }}(X)$. We accomplish this by embedding $\mathcal{T}_{\text {cov }}(X)$ in a certain twisted semigroup crossed product $B_{P} \rtimes_{\tau, X} P$ (Theorem 6.3 ), and then characterizing its faithful representations (Theorem 7.2). When $P=\mathbb{N}, \mathcal{T}_{\text {cov }}(X)$ is precisely the Toeplitz algebra of the Hilbert bimodule $X_{1}$ (the fiber over $1 \in \mathbb{N}$ ), and our Theorem 7.2 reduces to [13, Theorem 2.1]. In fact, the analysis in [13] was motivated by our preliminary work on this paper. We would like to point out in particular how the stronger result [13, Theorem 3.1] arose from our investigations into product systems, for it serves as a good illustration of the usefulness of Nica covariance. Suppose $Z$ is an orthogonal direct sum $\bigoplus_{\lambda \in \Lambda} Z^{\lambda}$ of Hilbert bimodules. Let $G$ be the free group on $\Lambda$, let $P$ be the subsemigroup of $G$ generated by $\Lambda$, and let $X$ be the unique product system over $P$ whose fiber over $\lambda$ is $Z^{\lambda}$. Then $\mathcal{T}_{\text {cov }}(X)$ is canonically isomorphic to the Toeplitz algebra of the bimodule $Z$, and [13, Theorem 3.1] follows from our Theorem 7.2. 
The main application of [13, Theorem 3.1] was to establish the simplicity of the graph algebras associated with certain infinite directed graphs [13, Corollary 4.3]. Although here we confine our applications to twisted semigroup crossed products, we anticipate that our results will also give interesting information about $\mathcal{O}_{X}$ when each of the fibers of $X$ arise from infinite directed graphs.

We begin in Section 1 by giving a brief review of Hilbert bimodules, their representations, and their $C^{*}$-algebras. In Section 2 we introduce product systems of Hilbert bimodules, discuss their representations, and define the algebras $\mathcal{T}_{X}$ and $\mathcal{O}_{X}$. In Section 3 we associate with each twisted semigroup dynamical system $(A, P, \beta, \omega)$ a product system $X=X(A, P, \beta, \omega)$ whose Cuntz-Pimsner algebra $\mathcal{O}_{X}$ is the twisted semigroup crossed product $A \rtimes_{\beta, \omega}$ $P$. We show that the Toeplitz algebra of $X(A, P, \beta, \omega)$ also has a crossed product structure, and this motivates the definition of a "Toeplitz" crossed product $\mathcal{T}\left(A \rtimes_{\beta, \omega} P\right)$ in which the endomorphisms are implemented not by isometries, but rather by partial isometries.

In Section 4 we generalize the notion of twisted crossed product by replacing the multiplier $\omega$ by a product system $X$ of Hilbert bimodules. This extends the philosophy developed in $[\mathbf{1 2}]$ that one should regard product systems as noncommutative cocycles. Hence given an action $\beta$ of $P$ as endomorphisms of a $C^{*}$-algebra $C$, we consider $(C, P, \beta, X)$ as a twisted semigroup dynamical system, and we define a twisted crossed product $C \rtimes_{\beta, X} P$.

In Section 5 we assume that $(G, P)$ is quasi-lattice ordered, and we discuss the notion of Nica covariance for a Toeplitz representation. As illustrated in [10, Example 1.3] using product systems of Hilbert spaces, when $(G, P)$ is not a total order it is possible that the $C^{*}$-algebra $\mathcal{T}_{\text {cov }}(X)$ which is "universal" for such representations may admit representations which are not the integrated form of a Nica-covariant Toeplitz representation. To avoid this pathology we adapt the methods of [10] to our setting: We define the notion of a product system being compactly aligned, and show that $\mathcal{T}_{\text {cov }}(X)$ is truly universal when $X$ is compactly aligned (Proposition 5.9). We show that $X$ is compactly aligned if the left action of $A$ on each fiber $X_{s}$ is by compact operators (Proposition 5.8); it follows that the product systems $X(A, P, \beta, \omega)$ associated with twisted semigroup dynamical systems are compactly aligned.

In Section 6 we consider a certain $C^{*}$-subalgebra $B_{P}$ of $\ell^{\infty}(P)$ which is invariant under left translation $\tau: P \rightarrow \operatorname{End}\left(\ell^{\infty}(P)\right)$. As in $[\mathbf{1 7}, \mathbf{1 2}]$, covariant representations of the twisted system $\left(B_{P}, P, \tau, X\right)$ are in one-one correspondence with Toeplitz representations of $X$ which are Nica-covariant (Proposition 6.1), and hence $\mathcal{T}_{\text {cov }}(X)$ embeds naturally as a subalgebra of $B_{P} \rtimes_{\tau, X} P$ (Theorem 6.3). When the left action of $A$ on each fiber $X_{s}$ is by compact operators, $\mathcal{T}_{\text {cov }}(X)$ is all of $B_{P} \rtimes_{\tau, X} P$.

In Section 7 we prove our main result, Theorem 7.2 , which characterizes the faithful representations of $B_{P} \rtimes_{\tau, X} P$ under the assumption that 
$X$ is compactly aligned and $\left(B_{P}, P, \tau, X\right)$ satisfies a certain amenability hypothesis. In Section 8 we give conditions on $(G, P)$ which ensure that $\left(B_{P}, P, \tau, X\right)$ is amenable. In particular, $\left(B_{P}, P, \tau, X\right)$ is amenable if $X$ is compactly aligned and $(G, P)$ is a free product $*\left(G^{\lambda}, P^{\lambda}\right)$ with each $G^{\lambda}$ amenable (Corollary 8.2).

Finally, in Section 9 we apply our Theorem 7.2 to the product system $X(A, P, \beta, \omega)$ of Section 3. When $(G, P)$ is a total order, $B_{P} \rtimes_{\tau, X} P$ is isomorphic to the Toeplitz crossed product $\mathcal{T}\left(A \rtimes_{\beta, \omega} P\right)$; in general $B_{P} \rtimes_{\tau, X} P$ is a certain quotient $\mathcal{T}_{\operatorname{cov}}\left(A \rtimes_{\beta, \omega} P\right)$ which also has a crossed product structure, and Theorem 9.3 characterizes its faithful representations. Applying this to the twisted system $\left(B_{P}, P, \tau, \omega\right)$, we show that $\mathcal{T}_{\text {cov }}\left(B_{P} \rtimes_{\tau, \omega} P\right)$ is universal for partial isometric representations of $P$ which are bicovariant (Proposition 9.6), and we obtain a characterization of its faithful representations (Theorem 9.7) which is particularly nice when $P$ is the free semigroup on infinitely many generators (Theorem 9.9).

The author thanks Iain Raeburn for the many helpful discussions while this research was being conducted.

\section{Preliminaries.}

Let $A$ be a separable $C^{*}$-algebra. A Hilbert bimodule over $A$ is a right Hilbert $A$-module $X$ together with a ${ }^{*}$-homomorphism $\phi: A \rightarrow \mathcal{L}(X)$ which is used to define a left action of $A$ on $X$ via $a \cdot x:=\phi(a) x$ for $a \in A$ and $x \in X$. A Toeplitz representation of $X$ in a $C^{*}$-algebra $B$ is a pair $(\psi, \pi)$ consisting of a linear map $\psi: X \rightarrow B$ and a homomorphism $\pi: A \rightarrow B$ such that

$$
\begin{aligned}
\psi(x \cdot a) & =\psi(x) \pi(a), \\
\psi(x)^{*} \psi(y) & =\pi\left(\langle x, y\rangle_{A}\right), \text { and } \\
\psi(a \cdot x) & =\pi(a) \psi(x)
\end{aligned}
$$

for $x, y \in X$ and $a \in A$. Given such a representation, there is homomorphism $\pi^{(1)}: \mathcal{K}(X) \rightarrow B$ which satisfies

$$
\pi^{(1)}\left(\Theta_{x, y}\right)=\psi(x) \psi(y)^{*} \quad \text { for all } x, y \in X,
$$

where $\Theta_{x, y}(z):=x \cdot\langle y, z\rangle_{A}$ for $z \in X$; see [22, p. 202], [16, Lemma 2.2], and $[\mathbf{1 3}$, Remark 1.7] for details. We say that $(\psi, \pi)$ is Cuntz-Pimsner covariant if

$$
\pi^{(1)}(\phi(a))=\pi(a) \quad \text { for all } a \in \phi^{-1}(\mathcal{K}(X)) .
$$

The Toeplitz algebra of $X$ is the $C^{*}$-algebra $\mathcal{T}_{X}$ which is universal for Toeplitz representations of $X[\mathbf{2 2}, \mathbf{1 3}]$, and the Cuntz-Pimsner algebra of $X$ is the $C^{*}$-algebra $\mathcal{O}_{X}$ which is universal for Toeplitz representations which are Cuntz-Pimsner covariant $[\mathbf{2 2}, \mathbf{9}, \mathbf{1 6}, \mathbf{1 8}, \mathbf{1 9}, \mathbf{1 1}]$. 
Every right Hilbert $A$-module $X$ is essential, in the sense that $X$ is the closed linear span of elements $x \cdot a$. We say that a Hilbert bimodule $X$ is essential if it is also essential as a left $A$-module; that is, if

$$
X=\overline{\operatorname{span}}\{\phi(a) x: a \in A, x \in X\} .
$$

When $X$ is essential, two applications of the Hewitt-Cohen Factorization Theorem allow us to write any $x \in X$ as $\phi(a) y \cdot b$ for some $y \in X$ and $a, b \in A$. Hence if $\left(a_{i}\right)$ is an approximate identity for $A$, then

$$
\left\|x-x \cdot a_{i}\right\| \rightarrow 0 \quad \text { and } \quad\left\|x-\phi\left(a_{i}\right) x\right\| \rightarrow 0 \quad \text { for all } x \in X \text {. }
$$

\section{Product systems of Hilbert bimodules.}

For each $n \geq 1$ the $n$-fold internal tensor product $X^{\otimes n}:=X \otimes_{A} \cdots \otimes_{A} X$ has a natural structure as a Hilbert bimodule over $A$; see [19, Section 2.2] for details. The following definition, based on Arveson's continuous tensor product systems over $(0, \infty)[\mathbf{3}]$, generalizes the collection $\left\{X^{\otimes n}: n \in \mathbb{N}\right\}$ to semigroups other than $\mathbb{N}$.

Definition 2.1. Suppose $P$ is a countable semigroup with identity $e$ and $p: X \rightarrow P$ is a family of Hilbert bimodules over $A$. Write $X_{s}$ for the fibre $p^{-1}(s)$ over $s \in P$, and write $\phi_{s}: A \rightarrow \mathcal{L}\left(X_{s}\right)$ for the homomorphism which defines the left action of $A$ on $X_{s}$. We say that $X$ is a (discrete) product system over $P$ if $X$ is a semigroup, $p$ is a semigroup homomorphism, and for each $s, t \in P \backslash\{e\}$ the map $(x, y) \in X_{s} \times X_{t} \mapsto x y \in X_{s t}$ extends to an isomorphism of the Hilbert bimodules $X_{s} \otimes_{A} X_{t}$ and $X_{s t}$. We also require that $X_{e}=A$ (with its usual right Hilbert module structure and $\phi_{e}(a) b=a b$ for $a, b \in A$ ), and that the multiplications $X_{e} \times X_{s} \rightarrow X_{s}$ and $X_{s} \times X_{e} \rightarrow X_{s}$ satisfy

$$
a x=\phi_{s}(a) x, \quad x a=x \cdot a \quad \text { for } a \in X_{e} \text { and } x \in X_{s} .
$$

Remark 2.2. Multiplication $X_{e} \times X_{s} \rightarrow X_{s}$ will not induce an isomorphism $X_{e} \otimes_{A} X_{s} \rightarrow X_{s}$ unless $X_{s}$ is essential as a left $A$-module.

Remark 2.3. The associativity of multiplication in $X$ implies that $\phi_{s t}(a)=$ $\phi_{s}(a) \otimes_{A} 1^{t}$ for all $a \in A$; that is, $\phi_{s t}(a)(x y)=\left(\phi_{s}(a) x\right) y$ for all $x \in X_{s}$ and $y \in X_{t}$.

Remark 2.4. It is possible that some of the $X_{s}$ may be zero.

Definition 2.5. Suppose $B$ is a $C^{*}$-algebra and $\psi: X \rightarrow B$; write $\psi_{s}$ for the restriction of $\psi$ to $X_{s}$. We call $\psi$ a Toeplitz representation of $X$ if:

(1) For each $s \in P,\left(\psi_{s}, \psi_{e}\right)$ is a Toeplitz representation of $X_{s}$; and

(2) $\psi(x y)=\psi(x) \psi(y)$ for $x, y \in X$.

If in addition each $\left(\psi_{s}, \psi_{e}\right)$ is Cuntz-Pimsner covariant, we say that $\psi$ is Cuntz-Pimsner covariant. 
Remark 2.6. By [13, Remark 1.1], every Toeplitz representation $\psi$ is contractive; moreover, if the homomorphism $\psi_{e}: A \rightarrow B$ is isometric, then so is $\psi$. Also, since we are assuming (2.1), a map $\psi: X \rightarrow B$ is a Toeplitz representation if it satisfies both (2) and

$$
\psi_{s}(x)^{*} \psi_{s}(y)=\psi_{e}\left(\langle x, y\rangle_{A}\right) \text { whenever } s \in P \text { and } x, y \in X_{s} .
$$

Notation 2.7. We write $\psi^{(s)}$ for the homomorphism of $\mathcal{K}\left(X_{s}\right)$ into $B$ which corresponds to the pair $\left(\psi_{s}, \psi_{e}\right)$, as in (1.1); that is,

$$
\psi^{(s)}\left(\Theta_{x, y}\right)=\psi_{s}(x) \psi_{s}(y)^{*} \quad \text { for all } x, y \in X_{s} .
$$

The Fock representation. Let $F(X)$ be the right Hilbert $A$-module

$$
F(X):=\bigoplus_{s \in P} X_{s}
$$

By this we mean the following: As a set, $F(X)$ is the subset of $\prod_{s \in P} X_{s}$ consisting of all elements $\left(x_{s}\right)$ for which $\sum_{s \in P}\left\langle x_{s}, x_{s}\right\rangle_{A}$ is summable in $A$; that is, for which $\sum_{s \in F}\left\langle x_{s}, x_{s}\right\rangle_{A}$ converges in norm as $F$ increases over the finite subsets of $P$. We write $\oplus x_{s}$ for $\left(x_{s}\right)$ to indicate that the above series is summable. The right action of $A$ is given by $\left(\oplus x_{s}\right) \cdot a:=\oplus\left(x_{s} \cdot a\right)$, and the inner product by $\left\langle\oplus x_{s}, \oplus y_{s}\right\rangle_{A}:=\sum_{s \in P}\left\langle x_{s}, y_{s}\right\rangle_{A}$. The algebraic direct sum $\bigodot_{s \in P} X_{s}$ is dense in $F(X)$.

Suppose $P$ is left-cancellative. Then for any $x \in X$ and $\oplus x_{t} \in F(X)$ we have $p\left(x x_{s}\right)=p\left(x x_{t}\right)$ if and only if $s=t$, so there is an element $\left(y_{s}\right) \in \prod X_{s}$ such that

$$
y_{s}= \begin{cases}x x_{t} & \text { if } s=p(x) t \\ 0 & \text { if } s \notin p(x) P\end{cases}
$$

we write $\left(x x_{t}\right)$ for $\left(y_{s}\right)$. Since $\left\langle x x_{s}, x x_{s}\right\rangle_{A} \leq\|x\|^{2}\left\langle x_{s}, x_{s}\right\rangle_{A}$ for each $s \in P$, the series $\sum\left\langle x x_{s}, x x_{s}\right\rangle_{A}$ is summable. It is routine to check that

$$
l(x)\left(\oplus x_{s}\right):=\oplus x x_{s} \quad \text { for } \oplus x_{s} \in F(X)
$$

determines an adjointable operator $l(x)$ on $F(X)$; indeed, the adjoint $l(x)^{*}$ is zero on any summand $X_{s}$ for which $s \notin p(x) P$, and on $X_{p(x) t}=\overline{\operatorname{span}} X_{p(x)} X_{t}$ it is determined by the formula $l(x)^{*}(y z)=\langle x, y\rangle_{A} \cdot z$ for $y \in X_{p(x)}$ and $z \in X_{t}$. It follows that $l: X \rightarrow \mathcal{L}(F(X))$ is a Toeplitz representation, called the Fock representation of $X$. The homomorphism $l_{e}: A \rightarrow \mathcal{L}(F(X))$ is simply the diagonal left action of $A$; that is, $l_{e}(a)=\oplus \phi_{s}(a)$. Since $\phi_{e}$ is just left multiplication on $X_{e}=A$, it is isometric, and hence so is $l_{e}$; by Remark 2.6, $l$ is isometric.

Proposition 2.8. Let $X$ be a product system over $P$ of Hilbert $A-A$ bimodules. Then there is a $C^{*}$-algebra $\mathcal{T}_{X}$, called the Toeplitz algebra of $X$, and a Toeplitz representation $i_{X}: X \rightarrow \mathcal{T}_{X}$, such that 
(a) for every Toeplitz representation $\psi$ of $X$, there is a homomorphism $\psi_{*}$ of $\mathcal{T}_{X}$ such that $\psi_{*} \circ i_{X}=\psi$; and

(b) $\mathcal{T}_{X}$ is generated as a $C^{*}$-algebra by $i_{X}(X)$.

The pair $\left(\mathcal{T}_{X}, i_{X}\right)$ is unique up to canonical isomorphism, and $i_{X}$ is isometric.

Proof. It is straightforward to translate the proof of [13, Proposition 1.3] to this setting.

Proposition 2.9. Let $X$ be a product system over $P$ of Hilbert $A-A$ bimodules. Then there is a $C^{*}$-algebra $\mathcal{O}_{X}$, called the Cuntz-Pimsner algebra of $X$, and a Toeplitz representation $j_{X}: X \rightarrow \mathcal{O}_{X}$ which is Cuntz-Pimsner covariant, such that

(a) for every Cuntz-Pimsner covariant Toeplitz representation $\psi$ of $X$, there is a homomorphism $\psi_{*}$ of $\mathcal{O}_{X}$ such that $\psi_{*} \circ j_{X}=\psi$; and

(b) $\mathcal{O}_{X}$ is generated as a $C^{*}$-algebra by $j_{X}(X)$.

The pair $\left(\mathcal{O}_{X}, j_{X}\right)$ is unique up to canonical isomorphism.

Remark 2.10. Although the universal map $i_{X}: X \rightarrow \mathcal{T}_{X}$ is always isometric, it is quite possible that $X$ might not admit any nontrivial Toeplitz representations which are Cuntz-Pimsner covariant, in which case $\mathcal{O}_{X}$ is trivial.

Proof of Proposition 2.9. With $\left(\mathcal{T}_{X}, i_{X}\right)$ as in Proposition 2.8, let $\mathcal{I}$ be the ideal in $\mathcal{T}_{X}$ generated by

$$
\left\{i_{X}(a)-i_{X}^{(s)}\left(\phi_{s}(a)\right): s \in P, a \in \phi_{s}^{-1}(\mathcal{K}(X))\right\} .
$$

Define $\mathcal{O}_{X}:=\mathcal{T}_{X} / \mathcal{I}$ and $j_{X}:=q \circ i_{X}$, where $q: \mathcal{T}_{X} \rightarrow \mathcal{O}_{X}$ is the canonical projection. Obviously $j_{X}$ is a Toeplitz representation which generates $\mathcal{O}_{X}$, and it is Cuntz-Pimsner covariant because $j_{X}^{(s)}=q \circ i_{X}^{(s)}$. If $\psi$ is another Cuntz-Pimsner covariant Toeplitz representation, then the homomorphism $\psi_{*}$ of $\mathcal{T}_{X}$ satisfies

$$
\psi_{*}\left(i_{X}(a)-i_{X}^{(s)}\left(\phi_{s}(a)\right)\right)=\psi(a)-\psi^{(s)}\left(\phi_{s}(a)\right)=0
$$

whenever $\phi_{s}(a) \in \mathcal{K}\left(X_{s}\right)$, and hence $\psi_{*}$ descends to the required homomorphism of $\mathcal{O}_{X}$ (also denoted $\left.\psi_{*}\right)$.

Proposition 2.11. Let $X$ be a product system over $\mathbb{N}$ of Hilbert $A-A$ bimodules. Then $\mathcal{T}_{X}$ is canonically isomorphic to the Toeplitz algebra $\mathcal{T}_{X_{1}}$ of the Hilbert bimodule $X_{1}$. If the left action on each fiber is isometric, or if the left action on each fiber is by compact operators, then $\mathcal{O}_{X}$ is canonically isomorphic to $\mathcal{O}_{X_{1}}$.

Proof. Let $i_{X}: X \rightarrow \mathcal{T}_{X}$ be universal for Toeplitz representations of $X$, and define $\mu:=\left(i_{X}\right)_{1}: X_{1} \rightarrow \mathcal{T}_{X}$ and $\pi:=\left(i_{X}\right)_{0}: A=X_{0} \rightarrow \mathcal{T}_{X}$. Since $(\mu, \pi)$ is a Toeplitz representation of $X_{1}$, we get a homomorphism $\mu \times \pi: \mathcal{T}_{X_{1}} \rightarrow \mathcal{T}_{X}$. 
To construct the inverse of $\mu \times \pi$, let $\left(i_{X_{1}}, i_{A}\right)$ be the universal Toeplitz representation of $X_{1}$ in $\mathcal{T}_{X_{1}}$, and fix $n \geq 1$. By [13, Proposition 1.8(1)], there is a linear map $\psi_{n}: X_{n} \rightarrow \mathcal{T}_{X_{1}}$ which satisfies

$$
\psi_{n}\left(x_{1} \cdots x_{n}\right):=i_{X_{1}}\left(x_{1}\right) \cdots i_{X_{1}}\left(x_{n}\right) \quad \text { for all } x_{1}, \ldots, x_{n} \in X_{1},
$$

and then $\left(\psi_{n}, i_{A}\right)$ is a Toeplitz representation of $X_{n}$. Defining $\psi_{0}:=i_{A}$ thus gives a Toeplitz representation $\psi: X \rightarrow \mathcal{T}_{X_{1}}$, and it is routine to check that $\psi_{*}: \mathcal{T}_{X} \rightarrow \mathcal{T}_{X_{1}}$ is the inverse of $\mu \times \pi$.

Now let $i_{X}: X \rightarrow \mathcal{O}_{X}$ be universal for Cuntz-Pimsner covariant Toeplitz representations of $X$. As above, we get a homomorphism $\mu \times \pi: \mathcal{O}_{X_{1}} \rightarrow \mathcal{O}_{X}$. To construct the inverse, we let $\left(i_{X_{1}}, i_{A}\right):\left(X_{1}, A\right) \rightarrow \mathcal{O}_{X_{1}}$ be universal and define a Toeplitz representation $\psi: X \rightarrow \mathcal{O}_{X_{1}}$ as before; we need to check that $\psi$ is Cuntz-Pimsner covariant under each of the hypotheses on the left action. By definition $\left(\psi_{1}, \psi_{0}\right)$ is Cuntz-Pimsner covariant, so we use induction. Assume that $\left(\psi_{n}, \psi_{0}\right)$ is Cuntz-Pimsner covariant for some $n \geq 1$, and suppose $a \in A$ acts compactly on the left of $X_{n+1}$; that is, $\phi(a) \otimes_{A} 1^{n} \in$ $\mathcal{K}\left(X_{n+1}\right)$. Since the left action is isometric on each fiber, by [11, Lemma 4.2] we have $\phi(a) \otimes_{A} 1^{n-1} \in \mathcal{K}\left(X_{n}\right)$; hence $\psi^{(n)}\left(\phi(a) \otimes_{A} 1^{n-1}\right)=\psi_{0}(a)$. But $[\mathbf{1 1}$, Lemma 4.5] gives $\psi^{(n+1)}\left(\phi(a) \otimes_{A} 1^{n}\right)=\psi^{(n)}\left(\phi(a) \otimes_{A} 1^{n-1}\right)$, so $\left(\psi_{n+1}, \psi_{0}\right)$ is Cuntz-Pimsner covariant.

Now suppose that $A$ acts by compact operators on each fiber. By representing $\mathcal{O}_{X_{1}}$ faithfully on a Hilbert space $\mathcal{H}$ we can assume that $\psi$ is a Toeplitz representation of $X$ on $\mathcal{H}$. Assuming again that $\left(\psi_{n}, \psi_{0}\right)$ is Cuntz-Pimsner covariant for some $n \geq 1$, [11, Lemma 1.9] gives $\overline{\psi_{0}(A) \mathcal{H} \subseteq}$ $\overline{\operatorname{span}}\left(\psi_{n}\left(X_{n}\right) \mathcal{H}\right)$. Let $x \in X_{n}$, and express $x=y \cdot a$ with $y \in X_{n}$ and $a \in A$. Since $\left(\psi_{1}, \psi_{0}\right)$ is Cuntz-Pimsner covariant and $\phi(a) \in \mathcal{K}\left(X_{1}\right)$, we have

$$
\psi_{n}(x)=\psi_{n}(y) \psi_{0}(a)=\psi_{n}(y) \psi^{(1)}(\phi(a)) .
$$

Now $\phi(a)$ can be approximated by a finite sum $\sum \Theta_{x_{i}, y_{i}}$, hence $\psi_{n}(x)$ can be approximated by a finite sum $\psi_{n}(y) \psi\left(x_{i}\right) \psi\left(y_{i}\right)^{*}=\psi_{n+1}\left(y x_{i}\right) \psi\left(y_{i}\right)^{*}$. Thus

$$
\overline{\psi_{0}(A) \mathcal{H}} \subseteq \overline{\operatorname{span}}\left(\psi_{n}\left(X_{n}\right) \mathcal{H}\right) \subseteq \overline{\operatorname{span}}\left(\psi_{n+1}\left(X_{n+1}\right) \mathcal{H}\right),
$$

and $\left(\psi_{n+1}, \psi_{0}\right)$ is Cuntz-Pimsner covariant by [11, Lemma 1.9].

Definition 2.12. Let $X$ be a product system over $P$ of Hilbert $A-A$ bimodules. A Toeplitz representation $\psi: X \rightarrow B$ is nondegenerate if the induced homomorphism $\psi_{*}: \mathcal{T}_{X} \rightarrow B$ is nondegenerate.

Lemma 2.13. Suppose each fiber $X_{s}$ is essential as a left A-module. Then a Toeplitz representation $\psi: X \rightarrow B$ is nondegenerate if and only if the homomorphism $\psi_{e}: A \rightarrow B$ is nondegenerate.

Proof. Let $\left(a_{i}\right)$ be an approximate identity for $A=X_{e}$. By $(1.2), i_{X}\left(a_{i}\right)$ is an approximate identity for $\mathcal{T}_{X}$, and the result follows. 


\section{Crossed products twisted by multipliers.}

Our main examples of product systems come from $C^{*}$-dynamical systems. Suppose $\beta$ is an action of $P$ as endomorphisms of $A$ such that $\beta_{e}$ is the identity endomorphism. We will assume that each $\beta_{s}$ is extendible; that is, that each $\beta_{s}$ extends to a strictly continuous endomorphism $\overline{\beta_{s}}$ of $M(A)$. For $P$ the positive cone of a totally ordered abelian group, Adji has shown that extendibility is necessary to define a reasonable crossed product $A \rtimes_{\beta} P$ [1].

In this section we will consider crossed products which are twisted by a multiplier $\omega$ of $P$; that is, by a function $\omega: P \times P \rightarrow \mathbb{T}$ which satisfies $\omega(e, e)=1$ and

$$
\omega(r, s) \omega(r s, t)=\omega(r, s t) \omega(s, t) \quad \text { for all } r, s, t \in P .
$$

We call $(A, P, \beta, \omega)$ a twisted semigroup dynamical system.

Definition 3.1. Let $B$ be a $C^{*}$-algebra. A function $V: P \rightarrow B$ is called an $\omega$-representation of $P$ if

$$
V_{s} V_{t}=\omega(s, t) V_{s t} \quad \text { for all } s, t \in P .
$$

If in addition each $V_{s}$ is an isometry (resp. partial isometry), $V$ is called isometric (resp. partial isometric) $\omega$-representation. A covariant representation of $(A, P, \beta, \omega)$ on a Hilbert space $\mathcal{H}$ is a pair $(\pi, V)$ consisting of a nondegenerate representation $\pi: A \rightarrow B(\mathcal{H})$ and an isometric $\omega$-representation $V: P \rightarrow B(\mathcal{H})$ such that

$$
\pi\left(\beta_{s}(a)\right)=V_{s} \pi(a) V_{s}^{*} \quad \text { for all } s \in P \text { and } a \in A .
$$

A crossed product for $(A, P, \beta, \omega)$ is a triple $\left(B, i_{A}, i_{P}\right)$ consisting of a $C^{*}$-algebra $B$, a nondegenerate homomorphism $i_{A}: A \rightarrow B$, and a map $i_{P}: P \rightarrow$ $M(B)$ such that

(a) if $\sigma$ is a nondegenerate representation of $B$, then $\left(\sigma \circ i_{A}, \bar{\sigma} \circ i_{P}\right)$ is a covariant representation of $(A, P, \beta, \omega)$;

(b) for every covariant representation $(\pi, V)$, there is a representation $\pi \times V$ such that $(\pi \times V) \circ i_{A}=\pi$ and $\overline{\pi \times V} \circ i_{P}=V$; and

(c) $B$ is generated as a $C^{*}$-algebra by $\left\{i_{A}(a) i_{P}(s): a \in A, s \in P\right\}$.

After establishing the existence of a crossed product, it is easily seen to be unique up to canonical isomorphism; we denote the crossed product algebra $A \rtimes_{\beta, \omega} P$.

We will construct a product system $X=X(A, P, \beta, \omega)$ over the opposite semigroup $P^{o}$, and show that its Cuntz-Pimsner algebra $\mathcal{O}_{X}$ is a crossed product for $(A, P, \beta, \omega)$. Moreover, we will show that the Toeplitz algebra of this product system also has a crossed product structure: It will be universal 
for pairs $(\pi, V)$ satisfying (3.2) in which $\pi$ is a nondegenerate representation of $A$ and $V$ is a partial isometric $\omega$-representation such that

$$
V_{s}^{*} V_{s} \pi(a)=\pi(a) V_{s}^{*} V_{s} \text { for all } s \in P \text { and } a \in A .
$$

We call such a pair $(\pi, V)$ a Toeplitz covariant representation of $(A, P, \beta, \omega)$, and write $\mathcal{T}\left(A \rtimes_{\beta, \omega} P\right)$ for the corresponding universal $C^{*}$-algebra, called the Toeplitz crossed product of $(A, P, \beta, \omega)$.

For each $s \in P$ let

$$
X_{s}:=\{s\} \times \overline{\beta_{s}}(1) A,
$$

and give $X_{s}$ the structure of a Hilbert bimodule over $A$ via

$$
(s, x) \cdot a:=(s, x a), \quad\langle(s, x),(s, y)\rangle_{A}:=x^{*} y,
$$

and

$$
\phi_{s}(a)(s, x):=\left(s, \beta_{s}(a) x\right) .
$$

Let $X=\bigsqcup_{s \in P} X_{s}$, let $p(s, x):=s$, and define multiplication in $X$ by

$$
(s, x)(t, y):=\left(t s, \overline{\omega(t, s)} \beta_{t}(x) y\right) \quad \text { for } x \in \overline{\beta_{s}}(1) A \text { and } y \in \overline{\beta_{t}}(1) A \text {. }
$$

Lemma 3.2. $X=X(A, P, \beta, \omega)$ is a product system over the opposite semigroup $P^{o}$. For each $s \in P$, the fiber $X_{s}$ is essential as a left $A$-module, and the left action of $A$ on $X_{s}$ is by compact operators.

Proof. Let $(s, x) \in X_{s}$ and $(t, y) \in X_{t}$. If $x=\overline{\beta_{s}}(1) a$ and $y=\overline{\beta_{t}}(1) b$, then

$$
\beta_{t}(x) y=\beta_{t}\left(\overline{\beta_{s}}(1) a\right) \overline{\beta_{t}}(1) b=\overline{\beta_{t}}\left(\overline{\beta_{s}}(1)\right) \beta_{t}(a) b=\overline{\beta_{t s}}(1) \beta_{t}(a) b,
$$

and hence the product $(s, x)(t, y)$ belongs to $X_{t s}$. Letting $a$ vary over an approximate identity for $A$, this product converges in norm to $\overline{\beta_{t s}}(1) b$, so the set of products $(s, x)(t, y)$ has dense linear span in $X_{t s}$. Hence to see that multiplication induces an isomorphism $X_{s} \otimes_{A} X_{t} \rightarrow X_{t s}$, it suffices to check that it preserves the inner product of any pair of elementary tensors:

$$
\begin{aligned}
\left\langle(s, x) \otimes_{A}(t, y),\right. & \left.\left(s, x^{\prime}\right) \otimes_{A}\left(t, y^{\prime}\right)\right\rangle_{A} \\
& =\left\langle(t, y), \phi_{t}\left(\left\langle(s, x),\left(s, x^{\prime}\right)\right\rangle_{A}\right)\left(t, y^{\prime}\right)\right\rangle_{A} \\
& =\left\langle(t, y), \phi_{t}\left(x^{*} x^{\prime}\right)\left(t, y^{\prime}\right)\right\rangle_{A} \\
& =\left\langle(t, y),\left(t, \beta_{t}\left(x^{*} x^{\prime}\right) y^{\prime}\right)\right\rangle_{A} \\
& =y^{*} \beta_{t}\left(x^{*} x^{\prime}\right) y^{\prime} \\
& =\left\langle\left(t s, \overline{\omega(t, s)} \beta_{t}(x) y\right),\left(t s, \overline{\omega(t, s)} \beta_{t}\left(x^{\prime}\right) y^{\prime}\right)\right\rangle_{A} \\
& =\left\langle(s, x)(t, y),\left(s, x^{\prime}\right)\left(t, y^{\prime}\right)\right\rangle_{A} .
\end{aligned}
$$


Multiplication is associative since

$$
\begin{aligned}
((s, x)(t, y))(r, z) & =\left(t s, \overline{\omega(t, s)} \beta_{t}(x) y\right)(r, z) \\
& =\left(r t s, \overline{\omega(r, t s)} \beta_{r}\left(\overline{\omega(t, s)} \beta_{t}(x) y\right) z\right) \\
& =\left(r t s, \overline{\omega(r t, s)} \beta_{r t}(x) \overline{\omega(r, t)} \beta_{r}(y) z\right) \\
& =(s, x)\left(r t, \overline{\omega(r, t)} \beta_{r}(y) z\right) \\
& =(s, x)((t, y)(r, z)) .
\end{aligned}
$$

If $\left(a_{i}\right)$ is an approximate identity for $A$, then for each $a \in A$ and $s \in P$ we have $\lim \phi\left(a_{i}\right)\left(s, \overline{\beta_{s}}(1) a\right)=\lim \left(s, \beta_{s}\left(a_{i}\right) a\right)=\left(s, \overline{\beta_{s}}(1) a\right)$, so each $X_{s}$ is essential. If $a \in A$, then by writing $a=b c^{*}$ with $b, c \in A$, we see that $\phi_{s}(a)=\Theta_{\left(s, \beta_{s}(b)\right),\left(s, \beta_{s}(c)\right)} \in \mathcal{K}\left(X_{s}\right)$ is compact.

Lemma 3.3. Let $i_{X}: X \rightarrow \mathcal{T}_{X}$ be universal for Toeplitz representations of $X$, and let $\left(a_{i}\right)$ be an approximate identity for $A$. Then for each $s \in P$, $i_{X}\left(s, \beta_{s}\left(a_{i}\right)\right)$ converges strictly in $M \mathcal{T}_{X}$.

Proof. Since each fiber $X_{t}$ is essential, any vector $\xi \in X_{t}$ can be written in the form $\xi=\phi_{t}(a) \eta \cdot b$ with $a, b \in A$ and $\eta \in X_{t}$. But then $i_{X}(\xi)=$ $i_{X}(e, a) i_{X}(\eta) i_{X}(e, b)$, and since elements of the form $i_{X}(\xi)$ generate $\mathcal{T}_{X}$ as a $C^{*}$-algebra, the result follows from the calculations

$$
i_{X}\left(s, \beta_{s}\left(a_{i}\right)\right) i_{X}(e, a)=i_{X}\left(s, \beta_{s}\left(a_{i}\right) a\right) \rightarrow i_{X}\left(s, \overline{\beta_{s}}(1) a\right)
$$

and

$$
i_{X}(e, a) i_{X}\left(s, \beta_{s}\left(a_{i}\right)\right)=i_{X}\left(s, \beta_{s}\left(a a_{i}\right)\right) \rightarrow i_{X}\left(s, \beta_{s}(a)\right) .
$$

Define $i_{A}: A \rightarrow \mathcal{T}_{X}$ by $i_{A}(a):=i_{X}(e, a)$, and define $i_{P}: P \rightarrow M \mathcal{T}_{X}$ by $i_{P}(s):=\lim i_{X}\left(s, \beta_{s}\left(a_{i}\right)\right)^{*}$.

Proposition 3.4. $\mathcal{T}_{X}$ and $\mathcal{O}_{X}$ are canonically isomorphic to $A \rtimes_{\beta, \omega} P$ and $\mathcal{T}\left(A \rtimes_{\beta, \omega} P\right)$, respectively. More precisely, $\left(\mathcal{T}_{X}, i_{A}, i_{P}\right)$ is a Toeplitz crossed product for $(A, P, \beta, \omega)$, and, with $q: \mathcal{T}_{X} \rightarrow \mathcal{O}_{X}$ the canonical projection, $\left(\mathcal{O}_{X}, q \circ i_{A}, \bar{q} \circ i_{P}\right)$ is a crossed product for $(A, P, \beta, \omega)$.

Proof. Taking $s=e$ in (3.4) and (3.5), shows that $i_{A}\left(a_{i}\right)$ converges strictly to the identity in $M\left(\mathcal{T}_{X}\right)$; that is, $i_{A}$ is nondegenerate. For Condition (a) of a Toeplitz crossed product, suppose $\sigma$ is a nondegenerate representation of $\mathcal{T}_{X}$; we must show that $(\pi, V):=\left(\sigma \circ i_{A}, \bar{\sigma} \circ i_{P}\right)$ is a Toeplitz covariant representation of $(A, P, \beta, \omega)$. First note that $\pi$ is nondegenerate since $\sigma$ and $i_{A}$ are. Equation (3.5) gives

$$
i_{A}(a) i_{P}(s)^{*}=i_{X}\left(s, \beta_{s}(a)\right) \quad \text { for all } a \in A \text { and } s \in P,
$$


SO

$$
\begin{aligned}
i_{P}(s) i_{A}(a) i_{P}(s)^{*} & =\lim i_{X}\left(s, \beta_{s}\left(a_{i}\right)\right)^{*} i_{X}\left(s, \beta_{s}(a)\right) \\
& =\lim i_{X}\left(e, \beta_{s}\left(a_{i}^{*} a\right)\right)=i_{X}\left(e, \beta_{s}(a)\right)=i_{A}\left(\beta_{s}(a)\right),
\end{aligned}
$$

and applying $\bar{\sigma}$ gives $V_{s} \pi(a) V_{s}^{*}=\pi\left(\beta_{s}(a)\right)$. In particular

$$
i_{P}(s) i_{P}(s)^{*}=\lim i_{P}(s) i_{A}\left(a_{i}\right) i_{P}(s)^{*}=\lim i_{A}\left(\beta_{s}\left(a_{i}\right)\right)=\overline{i_{A}}\left(\overline{\beta_{s}}(1)\right)
$$

is a projection, so $i_{P}(s)$, and hence $V_{s}$, is a partial isometry.

To establish (3.3), take any $a \in A$, write $a=b c^{*}$ with $b, c \in A$, and compute:

$$
\begin{aligned}
i_{A}\left(b c^{*}\right) i_{P}(s)^{*} i_{P}(s) & =\lim i_{X}\left(s, \beta_{s}\left(b c^{*}\right)\right) i_{X}\left(s, \beta_{s}\left(a_{i}\right)\right)^{*} \quad(\text { by }(3.6)) \\
& =\lim i_{X}\left(s, \beta_{s}(b)\right) i_{X}\left(e, \beta_{s}\left(c^{*}\right)\right) i_{X}\left(s, \beta_{s}\left(a_{i}\right)\right)^{*} \\
& =\lim i_{X}\left(s, \beta_{s}(b)\right)\left(i_{X}\left(s, \beta_{s}\left(a_{i}\right)\right) i_{X}\left(e, \beta_{s}(c)\right)\right)^{*} \\
& =\lim i_{X}\left(s, \beta_{s}(b)\right) i_{X}\left(s, \beta_{s}\left(a_{i} c\right)\right)^{*} \\
& =i_{X}\left(s, \beta_{s}(b)\right) i_{X}\left(s, \beta_{s}(c)\right)^{*}
\end{aligned}
$$

Taking adjoints, interchanging $b$ and $c$, and applying $\bar{\sigma}$ gives $V_{s}^{*} V_{s} \pi(a)=$ $\pi(a) V_{s}^{*} V_{s}$.

For every $s, t \in P$ we have

$$
\begin{aligned}
i_{P}(t)^{*} i_{P}(s)^{*} & =\left(\lim _{i} j_{X}\left(t, \beta_{t}\left(a_{i}\right)\right)\right)\left(\lim _{j} j_{X}\left(s, \beta_{s}\left(a_{j}\right)\right)\right) \\
& =\lim _{i} \lim _{j} j_{X}\left(s t, \overline{\omega(s, t)} \beta_{s}\left(\beta_{t}\left(a_{i}\right)\right) \beta_{s}\left(a_{j}\right)\right) \\
& =\lim _{i} j_{X}\left(s t, \overline{\omega(s, t)} \beta_{s t}\left(a_{i}\right)\right) \\
& =\overline{\omega(s, t)} i_{P}(s t)^{*} ;
\end{aligned}
$$

taking adjoints and applying $\bar{\sigma}$ gives $V_{s} V_{t}=\omega(s, t) V_{s t}$. This completes the proof of Condition (a).

For Condition (b), suppose $(\pi, V)$ is a Toeplitz covariant representation of $(A, P, \beta, \omega)$ on a Hilbert space $\mathcal{H}$. Define $\psi: X \rightarrow B(\mathcal{H})$ by

$$
\psi(s, x):=V_{s}^{*} \pi(x)
$$

Since $\pi$ is nondegenerate and $\pi(a)=\pi\left(\beta_{e}(a)\right)=V_{e} \pi(a) V_{e}^{*}$ for all $a \in A, V_{e}$ is a coisometry. Since $V_{e}^{2}=\omega(e, e) V_{e}=V_{e}$, we deduce that $V_{e}=1$. Thus

$$
\begin{aligned}
\psi(s, x)^{*} \psi(s, y) & =\pi(x)^{*} V_{s} V_{s}^{*} \pi(y)=\pi\left(x^{*} \overline{\beta_{s}}(1) y\right) \\
& =V_{e}^{*} \pi\left(x^{*} y\right) \quad\left(\text { since } y \in \overline{\beta_{s}}(1) A \text { and } V_{e}=1\right) \\
& =\psi\left(e, x^{*} y\right)=\psi\left(e,\langle(s, x),(s, y)\rangle_{A}\right),
\end{aligned}
$$


and since we also have

$$
\begin{array}{rlr}
\psi(s, x) \psi(t, y) & =V_{s}^{*} \pi(x) V_{t}^{*} \pi(y) & \\
& =V_{s}^{*} \pi(x) V_{t}^{*} V_{t} V_{t}^{*} \pi(y) \quad\left(V_{t}\right. \text { is a partial isometry) } \\
& =V_{s}^{*} V_{t}^{*} V_{t} \pi(x) V_{t}^{*} \pi(y) \\
& =\left(V_{t} V_{s}\right)^{*} \pi\left(\beta_{t}(x)\right) \pi(y) \\
& =\overline{\omega(t, s)} V_{t s}^{*} \pi\left(\beta_{t}(x) y\right) \\
& =\overline{\omega(t, s)} \psi\left(t s, \beta_{t}(x) y\right) \\
& =\psi((s, x)(t, y))
\end{array}
$$

$\psi$ is a Toeplitz representation of $X$. Let $\pi \times V$ be the representation $\psi_{*}$ : $\mathcal{T}_{X} \rightarrow B(\mathcal{H})$. Then

$$
(\pi \times V) \circ i_{A}(a)=\psi_{*} \circ i_{X}(e, a)=\psi(e, a)=V_{e}^{*} \pi(a)=\pi(a),
$$

and

$$
\begin{aligned}
\overline{\pi \times V} \circ i_{P}(s) \pi(a) & =\psi_{*}\left(i_{P}(s) i_{A}(a)\right) \\
& =\psi_{*}\left(i_{X}\left(s, \beta_{s}\left(a^{*}\right)\right)^{*}\right) \\
& =\psi\left(s, \beta_{s}\left(a^{*}\right)\right)^{*}=\pi\left(\beta_{s}(a)\right) V_{s} \\
& =V_{s} \pi(a) V_{s}^{*} V_{s}=V_{s} V_{s}^{*} V_{s} \pi(a)=V_{s} \pi(a)
\end{aligned}
$$

since $\pi$ is nondegenerate, this implies that $\overline{\pi \times V} \circ i_{P}=V$, as required. For Condition (c), simply note that $i_{A}(a) i_{P}(s)=i_{X}\left(s, \overline{\beta_{s}}(1) a^{*}\right)^{*}$, and elements of this form generate $\mathcal{T}_{X}$.

We now show that $\left(\mathcal{O}_{X}, q \circ i_{A}, \bar{q} \circ i_{P}\right)$ is a crossed product for $(A, P, \beta, \omega)$. Since $i_{A}$ and $q$ are nondegenerate, so is $q \circ i_{A}$. If $\rho$ is a nondegenerate representation of $\mathcal{O}_{X}$, then $\sigma:=\rho \circ q$ is a nondegenerate representation of $\mathcal{T}_{X}$. Hence $(\pi, V):=\left(\rho \circ q \circ i_{A}, \bar{\rho} \circ \bar{q} \circ i_{P}\right)=\left(\sigma \circ i_{A}, \bar{\sigma} \circ i_{P}\right)$ is a Toeplitz covariant representation of $(A, P, \beta, \omega)$. To see that each $V_{s}$ is an isometry, let $b, c \in A$. Since $q \circ i_{X}$ is Cuntz-Pimsner covariant, (3.7) gives

$$
\begin{aligned}
q \circ i_{A}\left(b c^{*}\right) \bar{q} \circ i_{P}(s)^{*} \bar{q} \circ i_{P}(s) & =q \circ i_{X}\left(s, \beta_{s}(b)\right) q \circ i_{X}\left(s, \beta_{s}(c)\right)^{*} \\
& =\left(q \circ i_{X}\right)^{(s)}\left(\Theta_{\left(s, \beta_{s}(b)\right),\left(s, \beta_{s}(c)\right)}\right) \\
& =\left(q \circ i_{X}\right)^{(s)}\left(\phi_{s}\left(b c^{*}\right)\right) \\
& =q \circ i_{X}\left(e, b c^{*}\right)=q \circ i_{A}\left(b c^{*}\right) .
\end{aligned}
$$

Since $q \circ i_{A}$ is nondegenerate, this implies that $\bar{q} \circ i_{P}(s)$, and hence $V_{s}$, is an isometry. This gives Condition (a) for a crossed product. Condition (c) is obvious, so it remains only to verify (b). Suppose $(\pi, V)$ is a covariant representation of $(A, P, \beta, \omega)$ on a Hilbert space $\mathcal{H}$, and define $\psi(s, x):=$ $V_{s}^{*} \pi(x)$ as before. We have already seen that $\psi$ is a Toeplitz representation 
of $X$, and it is Cuntz-Pimsner covariant since, for any $b, c \in A$,

$$
\begin{aligned}
\psi^{(s)}\left(\phi_{s}\left(b c^{*}\right)\right) & =\psi^{(s)}\left(\Theta_{\left(s, \beta_{s}(b)\right),\left(s, \beta_{s}(c)\right)}\right)=\psi\left(s, \beta_{s}(b)\right) \psi\left(s, \beta_{s}(c)\right)^{*} \\
& =V_{s}^{*} \pi\left(\beta_{s}(b)\right) \pi\left(\beta_{s}\left(c^{*}\right)\right) V_{s}=V_{s}^{*} V_{s} \pi\left(b c^{*}\right) V_{s}^{*} V_{s}=\psi\left(e, b c^{*} .\right.
\end{aligned}
$$

Defining $\pi \times V:=\psi_{*}: \mathcal{O}_{X} \rightarrow B(\mathcal{H})$ gives Condition (c).

\section{Crossed products twisted by product systems.}

Multipliers of $P$ correspond to product systems over $P$ of one-dimensional Hilbert spaces: Given a multiplier $\omega$, one defines multiplication on $P \times \mathbb{C}$ by $(s, z)(t, w):=(s t, \omega(s, t) z w)$. In this section we consider twisted semigroup dynamical systems in which the multiplier $\omega$ is replaced by a product system $X$ of Hilbert bimodules, and we construct a crossed product which is "twisted by $X$ ". For this, we first need to see how semigroups of endomorphism arise from Toeplitz representations of product systems.

Proposition 4.1. (1) Let $X$ be a Hilbert bimodule over $A$, and suppose $(\psi, \pi)$ is a Toeplitz representation of $X$ on a Hilbert space $\mathcal{H}$. Then there is a unique endomorphism $\alpha=\alpha^{\psi, \pi}$ of $\pi(A)^{\prime}$ such that

$$
\alpha(S) \psi(x)=\psi(x) S \quad \text { for all } S \in \pi(A)^{\prime} \text { and } x \in X,
$$

and such that $\alpha(1)$ vanishes on $(\psi(X) \mathcal{H})^{\perp}$.

(2) Let $X$ be a product system over $P$ of Hilbert $A-A$ bimodules in which each fiber $X_{s}$ is essential as a left A-module. Let $\psi$ be a nondegenerate Toeplitz representation of $X$ on a Hilbert space $\mathcal{H}$, and let $\alpha_{s}^{\psi}$ be the endomorphism $\alpha^{\psi_{s}, \psi_{e}}$ above. Then $\alpha^{\psi}: P \rightarrow \operatorname{End}\left(\psi_{e}(A)^{\prime}\right)$ is a semigroup homomorphism, and $\alpha_{e}^{\psi}$ is the identity endomorphism.

Proof. (1) The uniqueness of $\alpha$ is obvious. By [23, Proposition 2.69], there is a unital homomorphism $S \in \pi(A)^{\prime} \mapsto 1 \otimes_{A} S \in \operatorname{Ind} \pi(\mathcal{L}(X))^{\prime} \subseteq B\left(X \otimes_{A} \mathcal{H}\right)$ determined by

$$
1 \otimes_{A} S\left(x \otimes_{A} h\right)=x \otimes_{A} S h \quad \text { for } x \in X \text { and } h \in \mathcal{H} .
$$

Let $U: X \otimes_{A} \mathcal{H} \rightarrow \mathcal{H}$ be the isometry which satisfies $U\left(x \otimes_{A} h\right)=\psi(x) h$ (see the proof of [13, Proposition 1.6(1)]), and define

$$
\alpha(S):=U\left(1 \otimes_{A} S\right) U^{*} \quad \text { for all } S \in \pi(A)^{\prime} .
$$

Then $\alpha$ is a homomorphism, and $\alpha(1)=U U^{*}$ vanishes on $(\psi(X) \mathcal{H})^{\perp}$. If $S \in \pi(A)^{\prime}$ and $x \in X$, then for any $h \in \mathcal{H}$ we have

$$
\alpha(S) \psi(x) h=U\left(1 \otimes_{A} S\right)\left(x \otimes_{A} h\right)=U\left(x \otimes_{A} S h\right)=\psi(x) S h,
$$

giving (4.1).

Since $\pi(a) \psi(x) h=\psi(\phi(a) x) h$, the space $\overline{\operatorname{span}}\{\psi(x) h: x \in X, h \in \mathcal{H}\}$ reduces $\pi$; hence for any $S \in \pi(A)^{\prime}$ and $a \in A$, both $\alpha(S) \pi(a)$ and $\pi(a) \alpha(S)$ 
vanish on $(\psi(X) \mathcal{H})^{\perp}$. This and

$$
\begin{aligned}
\alpha(S) \pi(a) \psi(x) h & =\alpha(S) \psi(\phi(a) x) h=\psi(\phi(a) x) S h \\
& =\pi(a) \psi(x) S h=\pi(a) \alpha(S) \psi(x) h
\end{aligned}
$$

show that $\alpha\left(\pi(A)^{\prime}\right) \subseteq \pi(A)^{\prime}$.

(2) Let $s, t \in P$, and suppose $x \in X_{s}$ and $y \in X_{t}$. Vectors of the form $x y$ have dense linear span in $X_{s t}$; since $X_{t}$ is essential, this holds even when $s=e$ (see Remark 2.2). Since

$$
\begin{aligned}
\alpha_{s}^{\psi}\left(\alpha_{t}^{\psi}(S)\right) \psi_{s t}(x y) & =\alpha_{s}^{\psi}\left(\alpha_{t}^{\psi}(S)\right) \psi_{s}(x) \psi_{t}(y) \\
& =\psi_{s}(x) \alpha_{t}^{\psi}(S) \psi_{t}(y)=\psi_{s}(x) \psi_{t}(y) S=\psi_{s t}(x y) S,
\end{aligned}
$$

we deduce that

$$
\alpha_{s}^{\psi} \circ \alpha_{t}^{\psi}(S) \psi_{s t}(z)=\psi_{s t}(z) S \quad \text { for all } S \in \psi_{e}(A)^{\prime} \text { and } z \in X_{s t} .
$$

Once we show that $\alpha_{s}^{\psi} \circ \alpha_{t}^{\psi}(1)=\alpha_{s t}^{\psi}(1)$, it follows from the uniqueness of $\alpha_{s t}^{\psi}$ that $\alpha_{s}^{\psi} \circ \alpha_{t}^{\psi}=\alpha_{s t}^{\psi}$.

From (4.2) we see that $\alpha_{s}^{\psi} \circ \alpha_{t}^{\psi}(1) \geq \alpha_{s t}^{\psi}(1)$. Suppose that $\alpha_{s}^{\psi} \circ \alpha_{t}^{\psi}(1) f=f$; we will show that $\alpha_{s t}^{\psi}(1) f=f$, which will complete the proof. Since $f$ is in the range of $\alpha_{s}^{\psi}(1)$, it can be approximated by a finite sum $\sum_{i} \psi_{s}\left(x_{i}\right) g_{i}$. Then

$$
f \doteq \alpha_{s}^{\psi} \circ \alpha_{t}^{\psi}(1) \sum_{i} \psi_{s}\left(x_{i}\right) g_{i}=\sum_{i} \psi_{s}\left(x_{i}\right) \alpha_{t}^{\psi}(1) g_{i} .
$$

Now each $\alpha_{t}^{\psi}(1) g_{i}$ can be approximated by a finite sum $\sum_{j} \psi_{t}\left(y_{i j}\right) h_{i j}$, and then

$$
f \doteq \sum_{i, j} \psi_{s}\left(x_{i}\right) \psi_{t}\left(y_{i j}\right) h_{i j}=\sum_{i, j} \psi_{s t}\left(x_{i} y_{i j}\right) h_{i j} .
$$

Thus $f$ can be approximated arbitrarily closely by a vector in the range of $\alpha_{s t}^{\psi}(1)$, and hence $\alpha_{s t}^{\psi}(1) f=f$.

Since each $X_{t}$ is essential, the assumption that $\psi$ is nondegenerate implies that $\psi_{e}$ is a nondegenerate representation of $A$. Since $\alpha_{e}^{\psi}(S) \psi_{e}(a) h=$ $\psi_{e}(a) S h=S \psi_{e}(a) h$ for all $a \in A$ and $h \in \mathcal{H}$, we have $\alpha_{e}^{\psi}(S)=S$ for all $S \in \psi_{e}(A)^{\prime}$.

Consider a twisted semigroup dynamical system $(C, P, \beta, X)$ in which $C$ is a separable $C^{*}$-algebra, $\beta: P \rightarrow$ End $C$ is an action of the semigroup $P$ as extendible endomorphisms of $C$, and $X$ is a product system over $P$ of Hilbert $A-A$ bimodules. We assume that $\beta_{e}$ is the identity endomorphism, and that each fiber $X_{s}$ is essential as a left $A$-module.

Definition 4.2. A covariant representation of $(C, P, \beta, X)$ on a Hilbert space $\mathcal{H}$ is a pair $(L, \psi)$ consisting of a nondegenerate representation $L$ : 
$C \rightarrow B(\mathcal{H})$ and a nondegenerate Toeplitz representation $\psi: X \rightarrow B(\mathcal{H})$ such that

(i) $L(C) \subseteq \psi_{e}(A)^{\prime}$, and

(ii) $L \circ \beta_{s}=\alpha_{s}^{\psi} \circ L$ for $s \in P$.

Definition 4.3. A crossed product for $(C, P, \beta, X)$ is a triple $\left(B, i_{C}, i_{X}\right)$ consisting of a $C^{*}$-algebra $B$, a nondegenerate homomorphism $i_{C}: C \rightarrow$ $M(B)$, and a nondegenerate Toeplitz representation $i_{X}: X \rightarrow M(B)$ such that

(a) there is a faithful nondegenerate representation $\sigma$ of $B$ such that $(\bar{\sigma} \circ$ $\left.i_{C}, \bar{\sigma} \circ i_{X}\right)$ is a covariant representation of $(C, P, \beta, X)$;

(b) for every covariant representation $(L, \psi)$ of $(C, P, \beta, X)$, there is a representation $L \times \psi$ of $B$ such that $(\overline{L \times \psi}) \circ i_{C}=L$ and $(\overline{L \times \psi}) \circ i_{X}=\psi$; and

(c) the $C^{*}$-algebra $B$ is generated by $\left\{i_{C}(c) i_{X}(x): c \in C, x \in X\right\}$.

Remark 4.4. If each fiber $X_{s}$ has a finite basis $\left\{u_{s, 1}, \ldots, u_{s, n(s)}\right\}$ (in the sense that $x=\sum_{k} u_{s, k} \cdot\left\langle u_{s, k}, x\right\rangle_{A}$ for every $\left.x \in X_{s}\right)$, it is not hard to show that (a) is equivalent to asking that $i_{C}(c) i_{X}(a)=i_{X}(a) i_{C}(c)$ for all $c \in C$ and $a \in A=X_{e}$, and that

$$
i_{C}\left(\beta_{s}(c)\right)=\sum_{k} i_{X}\left(u_{s, k}\right) i_{C}(c) i_{X}\left(u_{s, k}\right)^{*} \quad \text { for all } s \in P \text { and } c \in C .
$$

In this case, $\left(\bar{\sigma} \circ i_{C}, \bar{\sigma} \circ i_{X}\right)$ will be a covariant representation of $(C, P, \beta, X)$ for every nondegenerate representation $\sigma$ of $B$; however, as demonstrated in [12, Example 2.5] for product systems of Hilbert spaces, in general one cannot expect this to be the case.

Proposition 4.5. If $(C, P, \beta, X)$ has a covariant representation, then it has a crossed product $\left(C \rtimes_{\beta, X} P, i_{C}, i_{X}\right)$ which is unique in the following sense: If $\left(B, i_{C}^{\prime}, i_{X}^{\prime}\right)$ is another crossed product for $(C, P, \beta, X)$, then there is an isomorphism $\theta: C \rtimes_{\beta, X} P \rightarrow B$ such that $\bar{\theta} \circ i_{C}=i_{C}^{\prime}$ and $\theta \circ i_{X}=i_{X}^{\prime}$.

Remark 4.6. When $X$ is the product system $P \times \mathbb{C}$ with multiplication given by a multiplier $\omega$, it is not hard to see that $C \rtimes_{\beta, X} P$ is precisely the crossed product $C \rtimes_{\beta, \omega} P$ defined in the previous section. If $C$ is unital and $A=\mathbb{C}$, then $C \rtimes_{\beta, X} P$ is the crossed product defined in [12, Section 2].

Proof of Proposition 4.5. If $S$ is a set of pairs $(L, \psi)$ consisting of maps $L: C \rightarrow B\left(\mathcal{H}_{L, \psi}\right)$ and $\psi: X \rightarrow B\left(\mathcal{H}_{L, \psi}\right)$, then $(\oplus L, \oplus \psi)$ is a covariant representation of $(C, P, \beta, X)$ if and only if each $(L, \psi)$ is. The main point here is that the value of $\alpha_{s}^{\oplus \psi}$ on an element of $(\oplus \psi)_{e}(A)^{\prime}$ of the form $\oplus L(c)$ is $\oplus \alpha_{s}^{\psi}(L(c))$. 
Suppose $(L, \psi)$ is a nondegenerate covariant representation on a separable Hilbert space $\mathcal{H}$; that is, the $C^{*}$-algebra

$$
\mathcal{U}:=C^{*}(\{L(c) \psi(x): c \in C, x \in X\})
$$

acts nondegenerately on $\mathcal{H}$. We will identify the multiplier algebra of $\mathcal{U}$ with the concrete $C^{*}$-algebra

$$
M(\mathcal{U})=\{S \in B(\mathcal{H}): S T, T S \in \mathcal{U} \text { for every } T \in \mathcal{U}\} .
$$

We claim that $L(C) \cup \psi(X) \subseteq M(\mathcal{U})$. For this, it suffices to check that multiplying a generator $L(c) \psi(x)$ of $\mathcal{U}$ on either the left or the right by an operator of the form $L(d), \psi(y)$, or $\psi(y)^{*}$ yields another element of $\mathcal{U}$. Certainly $L(d) L(c) \psi(x)=L(d c) \psi(x) \in \mathcal{U}$ and $L(c) \psi(x) \psi(y)=L(c) \psi(x y) \in$ $\mathcal{U}$, and since

$$
\psi(y) L(c)=\alpha_{p(y)}^{\psi}(L(c)) \psi(y)=L\left(\beta_{p(y)}(c)\right) \psi(y),
$$

we also have $\psi(y) L(c) \psi(x)=L\left(\beta_{p(y)}(c)\right) \psi(y x) \in \mathcal{U}$ and $L(c) \psi(x) L(d)=$ $L\left(c \beta_{p(x)}(d)\right) \psi(x) \in \mathcal{U}$. Writing $c=c_{1}^{*} c_{2}$ with $c_{1}, c_{2} \in C$ gives

$$
\psi(y)^{*} L(c) \psi(x)=\left(L\left(c_{1}\right) \psi(y)\right)^{*} L\left(c_{2}\right) \psi(x) \in \mathcal{U} .
$$

Finally, to see that $L(c) \psi(x) \psi(y)^{*} \in \mathcal{U}$, we use a trick from [1]. Let $\left(c_{i}\right)$ be an approximate identity for $C$; we claim that

$$
L(c) \psi(x) L\left(c_{i}\right) \stackrel{\|}{\longrightarrow} L(c) \psi(x) .
$$

Since $L$ is nondegenerate, $L(c) \psi(x) L\left(c_{i}\right)$ converges strongly to $L(c) \psi(x)$. On the other hand, using (4.3) we see that $L(c) \psi(x) L\left(c_{i}\right)=L\left(c \beta_{p(x)}\left(c_{i}\right)\right) \psi(x)$ converges in norm (to $L\left(c \overline{\beta_{p(x)}}(1)\right) \psi(x)$ ), and (4.4) follows. Hence

$$
L(c) \psi(x) L\left(c_{i}\right) \psi(y)^{*} \stackrel{\|}{\longrightarrow} L(c) \psi(x) \psi(y)^{*},
$$

and since

$$
L(c) \psi(x) L\left(c_{i}\right) \psi(y)^{*}=L(c) \psi(x) \psi(y)^{*} L\left(\beta_{p(y)}\left(c_{i}\right)\right) \in \mathcal{U},
$$

we deduce that $L(c) \psi(x) \psi(y)^{*} \in \mathcal{U}$.

Since $M(\mathcal{U}) \subseteq \mathcal{U}^{\prime \prime}$, we have shown in particular that the ranges of $L$ and $\psi$ are contained in $\mathcal{U}^{\prime \prime}$. Consequently, any decomposition $1=\sum Q_{\lambda}$ of the identity as a sum of mutually orthogonal projections $Q_{\lambda} \in \mathcal{U}^{\prime}$ gives corresponding decompositions $L=\oplus Q_{\lambda} L$ and $\psi=\oplus Q_{\lambda} \psi$, and by the first paragraph each pair $\left(Q_{\lambda} L, Q_{\lambda} \psi\right)$ is a covariant representation of $(C, P, \beta, X)$. By the usual Zorn's Lemma argument we can choose these projections such that $\mathcal{U}$ acts cyclically on $Q_{\lambda} \mathcal{H}$; since $C^{*}\left(\left\{Q_{\lambda} L(c) Q_{\lambda} \psi(x): c \in C, x \in X\right\}\right)=$ $Q_{\lambda} \mathcal{U}$ acts cyclically on $Q_{\lambda} \mathcal{H}$, this shows that every covariant representation of $(C, P, \beta, X)$ decomposes as a direct sum of cyclic representations.

Let $S$ be a set of cyclic covariant representations with the property that every cyclic covariant representation of $(C, P, \beta, X)$ is unitarily equivalent 
to an element in $S$. It can be shown that such a set $S$ exists by fixing a Hilbert space $\mathcal{H}$ of sufficiently large cardinality (depending on the cardinalities of $C$ and $X$ ) and considering only representations on $\mathcal{H}$. Note that $S$ is nonempty because the system has a covariant representation, which has a cyclic summand.

Define $i_{C}:=\bigoplus_{(L, \psi) \in S} L$ and $i_{X}:=\bigoplus_{(L, \psi) \in S} \psi$, and let $C \rtimes_{\beta, X} P$ be the $C^{*}$-algebra generated by $\left\{i_{C}(c) i_{X}(x): c \in C, x \in X\right\}$. By the first paragraph, $\left(i_{C}, i_{X}\right)$ is a covariant representation of $(C, P, \beta, X)$, and it is nondegenerate since each $(L, \psi)$ is. We deduce that both $i_{C}$ and $i_{X}$ map into $M\left(C \rtimes_{\beta, X} P\right)$, and that Condition (a) for a crossed product is satisfied by taking $\sigma$ to be the identity representation. Condition (c) is trivial, and (b) holds because every covariant representation decomposes as a direct sum of cyclic representations. We need to show that $i_{C}: C \rightarrow$ $M\left(C \rtimes_{\beta, X} P\right)$ and $i_{X}: X \rightarrow M\left(C \rtimes_{\beta, X} P\right)$ are nondegenerate. For this, let $c \in C$ and $x \in X$. If $\left(a_{i}\right)$ is an approximate identity for $A=X_{e}$, then by (1.2) we have $i_{C}(c) i_{X}(x) i_{X}\left(a_{i}\right)=i_{C}(c) i_{X}\left(x \cdot a_{i}\right) \rightarrow i_{C}(c) i_{X}(x)$ and $i_{X}\left(a_{i}\right) i_{C}(c) i_{X}(x)=i_{C}(c) i_{X}\left(a_{i}\right) i_{X}(x)=i_{C}(c) i_{X}\left(\phi\left(a_{i}\right) x\right) \rightarrow i_{C}(c) i_{X}(x)$, so $i_{X}$ is nondegenerate (Lemma 2.13). If $\left(c_{i}\right)$ is an approximate identity for $C$, then $i_{C}\left(c_{i}\right) i_{C}(c) i_{X}(x)=i_{C}\left(c_{i} c\right) i_{X}(x) \rightarrow i_{C}(c) i_{X}(x)$, and since $i_{C}$ is nondegenerate as a representation on Hilbert space, (4.4) gives $i_{C}(c) i_{X}(x) i_{C}\left(c_{i}\right) \rightarrow$ $i_{C}(c) i_{X}(x)$. Thus $i_{C}$ is nondegenerate.

For the uniqueness assertion, suppose $\left(B, i_{C}^{\prime}, i_{X}^{\prime}\right)$ is another crossed product. Condition (a) allows us to assume that $\left(i_{C}, i_{X}\right)$ and $\left(i_{C}^{\prime}, i_{X}^{\prime}\right)$ are covariant representations of $(C, P, \beta, X)$ on Hilbert spaces $\mathcal{H}$ and $\mathcal{H}^{\prime}$. Condition (b) then gives a representation $i_{C}^{\prime} \times i_{X}^{\prime}: C \rtimes_{\beta, X} P \rightarrow B\left(\mathcal{H}^{\prime}\right)$ whose image is contained in $B$ since $i_{C}^{\prime} \times i_{X}^{\prime}\left(i_{C}(c) i_{X}(x)\right)=i_{C}^{\prime}(c) i_{X}^{\prime}(x)$. Similarly one obtains a map $i_{C} \times i_{X}: B \rightarrow C \rtimes_{\beta, X} P$ which is obviously an inverse for $i_{C}^{\prime} \times i_{X}^{\prime}: C \rtimes_{\beta, X} P \rightarrow B$.

If $P$ is a subsemigroup of a group $G$, then there is a dual coaction of $G$ on $C \rtimes_{\beta, X} P$ :

Proposition 4.7. Suppose $(C, P, \beta, X)$ is a twisted system which has a covariant representation. If $P$ is a subsemigroup of a group $G$, then there is an injective coaction

$$
\delta: C \rtimes_{\beta, X} P \rightarrow\left(C \rtimes_{\beta, X} P\right) \otimes_{\min } C^{*}(G)
$$

such that

$$
\delta\left(i_{C}(c) i_{X}(x)\right)=i_{C}(c) i_{X}(x) \otimes i_{G}(p(x)) .
$$

If $G$ is abelian, there is a strongly continuous action $\widehat{\beta}$ of $\widehat{G}$ on $C \rtimes_{\beta, X} P$ such that

$$
\widehat{\beta}_{\gamma}\left(i_{C}(c) i_{X}(x)\right)=\gamma(p(x)) i_{C}(c) i_{X}(x)
$$


Proof. We follow [12, Proposition 2.7]. Let $\sigma$ be a faithful nondegenerate representation $\sigma$ of $C \rtimes_{\beta, X} P$ such that $(L, \psi):=\left(\bar{\sigma} \circ i_{C}, \bar{\sigma} \circ i_{X}\right)$ is a covariant representation of $(C, P, \beta, X)$, and let $U$ be a unitary representation of $G$ whose integrated form $\pi_{U}$ is faithful on $C^{*}(G)$. We claim that $(L \otimes 1, \psi \otimes$ $(U \circ p))$ is a covariant representation of $(C, P, \beta, X)$. Most of the verifications are routine, so we check only that

$$
L\left(\beta_{s}(c)\right) \otimes 1=\alpha_{s}^{\psi \otimes(U \circ p)}(L(c) \otimes 1) \quad \text { for all } s \in P \text { and } c \in C .
$$

For this, we show that $L\left(\beta_{s}(c)\right) \otimes 1$ satisfies the properties which characterize $\alpha_{s}^{\psi \otimes(U \circ p)}(L(c) \otimes 1)$ (Proposition 4.1). First, let $x \in X_{s}$; we show that (4.5) holds on any vector in the range of $(\psi \otimes(U \circ p))(x)=\psi_{s}(x) \otimes U_{s}$ :

$$
\begin{aligned}
\left(L\left(\beta_{s}(c)\right) \otimes 1\right)\left(\psi_{s}(x) \otimes U_{s}\right)=\alpha_{s}^{\psi}(L(c)) \psi_{s}(x) \otimes U_{s}=\psi_{s}(x) L(c) \otimes U_{s} \\
=\left(\psi_{s}(x) \otimes U_{s}\right)(L(c) \otimes 1)=\alpha_{s}^{\psi \otimes(U \circ p)}(L(c) \otimes 1)\left(\psi_{s}(x) \otimes U_{s}\right) .
\end{aligned}
$$

Next, note that $\alpha_{s}^{\psi \otimes(U \circ p)}(1)$ is the projection onto

$$
\begin{aligned}
\overline{\operatorname{span}}\{(\psi & \left.\otimes(U \circ p))(x) \xi: x \in X_{s}, \xi \in \mathcal{H}_{\sigma} \otimes \mathcal{H}_{U}\right\} \\
& =\overline{\operatorname{span}}\left\{\psi_{s}(x) h \otimes U_{s} k: x \in X_{s}, h \in \mathcal{H}_{\sigma}, k \in \mathcal{H}_{U}\right\} \\
& =\overline{\operatorname{span}}\left\{\psi_{s}(x) h: x \in X_{s}, h \in \mathcal{H}_{\sigma}\right\} \otimes \mathcal{H}_{U},
\end{aligned}
$$

which is precisely the range of $\alpha_{s}^{\psi}(1) \otimes 1$. Since $L\left(\beta_{s}(c)\right) \otimes 1=\alpha_{s}^{\psi}(L(c)) \otimes 1$ vanishes on the range of $1-\alpha_{s}^{\psi}(1) \otimes 1,(4.5)$ follows from the uniqueness assertion of Proposition 4.1.

Since $(L \otimes 1, \psi \otimes(U \circ p))$ is covariant, there is a representation $\rho$ of $C \rtimes_{\beta, X} P$ such that

$$
\begin{aligned}
\rho\left(i_{C}(c) i_{X}(x)\right) & =(L(c) \otimes 1)\left(\psi(x) \otimes U_{p(x)}\right) \\
& =\left(\sigma \otimes \pi_{U}\right)\left(i_{C}(c) i_{X}(x) \otimes i_{G}(p(x))\right) .
\end{aligned}
$$

Since $\sigma$ and $\pi_{U}$ are faithful, $\sigma \otimes \pi_{U}$ is faithful on $\left(C \rtimes_{\beta, X} P\right) \otimes_{\min } C^{*}(G)$, and we can define $\delta:=\left(\sigma \otimes \pi_{U}\right)^{-1} \circ \rho$.

By checking on generators it is easy to see that $\delta$ satisfies the coaction identity (id $\left.\otimes \delta_{G}\right) \circ \delta=(\delta \otimes \mathrm{id}) \circ \delta$, and $\delta$ is injective since $\sigma=(\sigma \otimes \epsilon) \circ \delta$, with $\epsilon$ the augmentation representation of $C^{*}(G)$ (i.e., $\epsilon\left(i_{G}(s)\right)=1$ for all $s \in G$ ). When $G$ is abelian, $\widehat{\beta}$ is the action canonically associated with $\delta$.

\section{Nica covariance.}

Now suppose $P$ is a subsemigroup of a group $G$ such that $P \cap P^{-1}=\{e\}$. Then $s \leq t$ iff $s^{-1} t \in P$ defines a partial order on $G$ which is left-invariant: For any $r, s, t \in P$ we have $s \leq t$ iff $r s \leq r t$. Following Nica [20], we say that $(G, P)$ is a quasi-lattice ordered group if every finite subset of $G$ which has an upper bound in $P$ has a least upper bound in $P$. When $s, t \in P$ have a common upper bound, we denote their least upper bound by $s \vee t$; when $s$ 
and $t$ have no common upper bound we write $s \vee t=\infty$. For a finite subset $C=\left\{t_{1}, \ldots, t_{n}\right\}$ of $P$, we write $\sigma C$ for $t_{1} \vee \cdots \vee t_{n}$.

Definition 5.1. Suppose $(G, P)$ is a quasi-lattice ordered group and $X$ is a product system over $P$ of essential Hilbert $A-A$ bimodules. We call a Toeplitz representation $\psi: X \rightarrow B(\mathcal{H})$ Nica covariant if

$$
\alpha_{s}^{\psi}(1) \alpha_{t}^{\psi}(1)= \begin{cases}\alpha_{s \vee t}^{\psi}(1) & \text { if } s \vee t<\infty \\ 0 & \text { otherwise. }\end{cases}
$$

Remark 5.2. If $(G, P)$ is totally ordered, then every Toeplitz representation of $X$ is Nica covariant.

Lemma 5.3. Let $l: X \rightarrow \mathcal{L}(F(X))$ be the Fock representation, and suppose $\pi$ is a representation of $A$ on a Hilbert space $\mathcal{H}$. Then

$$
\Psi:=F(X)-\operatorname{Ind}_{A} \mathcal{L}(F(X)) \pi \circ l
$$

is a Nica-covariant Toeplitz representation of $X$. If $\pi$ is faithful, then $\Psi$ is isometric.

Proof. Since $l$ is a Toeplitz representation, so is $\Psi$. Let $s \in P$. The range of $\alpha_{s}^{\Psi}(1)$ is

$$
\begin{aligned}
\overline{\operatorname{span}}\left\{\Psi(x) \xi: x \in X_{s}, \xi \in F(X) \otimes_{A} \mathcal{H}\right\} \\
\quad=\overline{\operatorname{span}}\left\{l(x) y \otimes_{A} h: x \in X_{s}, y \in F(X), h \in \mathcal{H}\right\}=\bigoplus_{s \leq r} X_{r} \otimes_{A} \mathcal{H} .
\end{aligned}
$$

Hence for any $s, t \in P$, the range of $\alpha_{s}^{\Psi}(1) \alpha_{t}^{\Psi}(1)$ is

$$
\left(\bigoplus_{s \leq r} X_{r} \otimes_{A} \mathcal{H}\right) \cap\left(\bigoplus_{t \leq r} X_{r} \otimes_{A} \mathcal{H}\right)
$$

which is $\bigoplus_{s \vee t \leq r} X_{r} \otimes_{A} \mathcal{H}=\operatorname{ran} \alpha_{s \vee t}^{\Psi}(1)$ if $s \vee t<\infty$, and is zero otherwise. If $\pi$ is faithful then so is $F(X)-\operatorname{Ind}_{A} \mathcal{L}(F(X)) \pi$; since $l$ is isometric, this implies that $\Psi$ is isometric.

Proposition 5.4. Let $(G, P)$ be a quasi-lattice ordered group such that every $s, t \in P$ have a common upper bound. Let $X$ be a product system over $P$ of essential Hilbert $A-A$ bimodules such that the left action of $A$ on each fiber $X_{s}$ is by compact operators. Then every Toeplitz representation $\psi: X \rightarrow B(\mathcal{H})$ which is Cuntz-Pimsner covariant is also Nica covariant.

Proof. Fix $s \in P$. Since $\left(\psi_{s}, \psi_{e}\right)$ is Cuntz-Pimsner covariant and $\phi_{s}(A) \subseteq$ $\mathcal{K}\left(X_{s}\right),\left[\mathbf{1 1}\right.$, Lemma 1.9] gives $\psi_{e}(A) \mathcal{H} \subseteq \overline{\operatorname{span}} \psi_{s}\left(X_{s}\right) \mathcal{H}$. But $X_{s}$ is essential, so the reverse inclusion holds as well, and since $\overline{\operatorname{span}} \psi_{s}\left(X_{s}\right) \mathcal{H}$ is precisely the range of $\alpha_{s}^{\psi}(1)$, we deduce that $\alpha_{s}^{\psi}(1)$ is constant in $s$. Since $s \vee t<\infty$ for all $s, t \in P$, this implies that $\psi$ is Nica covariant. 
There are product systems for which Nica covariance is not a $C^{*}$-algebraic condition; that is, if $\psi: X \rightarrow B(\mathcal{H})$ is Nica covariant and $\sigma: C^{*}(\psi(X)) \rightarrow$ $B(\mathcal{K})$ is a homomorphism, the composition $\sigma \circ \psi$ need not be Nica covariant [10, Example 1.3]. We pause a moment to show how to adapt the methods of [10] to avoid this pathology. The following Lemma collects some results we shall need for both this and the sequel.

Lemma 5.5. Suppose $X$ is a product system over $P$ of essential Hilbert $A-A$ bimodules, $\psi: X \rightarrow B(\mathcal{H})$ is a Toeplitz representation, and $s \in P$.

(1) There is a strict-strong continuous representation $\rho_{s}^{\psi}: \mathcal{L}\left(X_{s}\right) \rightarrow B(\mathcal{H})$ such that

$\rho_{s}^{\psi}(S) \psi_{s}(x) h=\psi_{s}(S x) h \quad$ for all $S \in \mathcal{L}\left(X_{s}\right), x \in X_{s}$, and $h \in \mathcal{H}$,

and such that $\rho_{s}^{\psi}(S)$ vanishes on $\left(\psi_{s}\left(X_{s}\right) \mathcal{H}\right)^{\perp}$. Moreover, $\rho_{s}^{\psi}(S)=$ $\psi^{(s)}(S)$ for every $S \in \mathcal{K}\left(X_{s}\right)$.

(2) $\rho_{s}^{\psi}(1)=\alpha_{s}^{\psi}(1)$.

(3) If $a \in A$ satisfies $\phi_{s}(a) \in \mathcal{K}\left(X_{s}\right)$, then

$$
\psi_{e}(a) \rho_{s}^{\psi}(1)=\psi^{(s)}\left(\phi_{s}(a)\right)=\rho_{s}^{\psi}(1) \psi_{e}(a) .
$$

(4) If $Q \in \psi_{e}(A)^{\prime}$, then $\alpha_{s}^{\psi}(Q) \in \rho_{s}^{\psi}\left(\mathcal{L}\left(X_{s}\right)\right)^{\prime}$. Further, if $Q$ is a projection such that $\psi_{e}$ acts faithfully on $Q \mathcal{H}$, then $\rho_{s}^{\psi}$ acts faithfully on $\alpha_{s}^{\psi}(Q) \mathcal{H}$.

(5) For all $S \in \mathcal{L}\left(X_{s}\right)$ and $t \in P$ we have

$$
\rho_{s t}^{\psi}\left(S \otimes_{A} 1\right)=\rho_{s}^{\psi}(S) \rho_{s t}^{\psi}(1)=\rho_{s t}^{\psi}(1) \rho_{s}^{\psi}(S),
$$

where $S \otimes_{A} 1(x y):=(S x) y$ for all $x \in X_{s}$ and $y \in X_{t}$.

(6) If $t \in P$ and $z, w \in X_{s}$, then $\rho_{s t}^{\psi}\left(\Theta_{z, w} \otimes_{A} 1\right)=\psi(z) \alpha_{t}^{\psi}(1) \psi(w)^{*}$.

Proof. (1) See [13, Proposition 1.6(1)]. For the continuity assertion, suppose $S_{\lambda} \rightarrow S$ strictly in $\mathcal{L}\left(X_{s}\right)=M \mathcal{K}\left(X_{s}\right), x \in X_{s}$, and $h \in \mathcal{H}$. There exists $K \in \mathcal{K}\left(X_{s}\right)$ and $y \in X_{s}$ such that $x=K y$, and then

$$
\rho_{s}^{\psi}\left(S_{\lambda}\right) \psi_{s}(x) h=\rho_{s}^{\psi}\left(S_{\lambda} K\right) \psi_{s}(y) h \rightarrow \rho_{s}^{\psi}(S K) \psi_{s}(y) h=\rho_{s}^{\psi}(S) \psi_{s}(x) h .
$$

(2) Both $\rho_{s}^{\psi}(1)$ and $\alpha_{s}^{\psi}(1)$ are the projection onto $\overline{\operatorname{span}}\left\{\psi_{s}\left(X_{s}\right) \mathcal{H}\right\}$.

(3) If $x \in X_{s}$ and $h \in \mathcal{H}$, then

$$
\psi_{e}(a) \rho_{s}^{\psi}(1) \psi_{s}(z) h=\psi_{e}(a) \psi_{s}(z) h=\psi_{s}\left(\phi_{s}(a) z\right) h=\psi^{(s)}\left(\phi_{s}(a)\right) \psi_{s}(z) h ;
$$

since both sides of (5.1) are supported on $\overline{\operatorname{span}} \psi_{s}\left(X_{s}\right) \mathcal{H}$, this implies that $\psi_{e}(a) \rho_{s}^{\psi}(1)=\psi^{(s)}\left(\phi_{s}(a)\right)$. By $(2), \rho_{s}^{\psi}(1)$ commutes with $\psi_{e}(a)$, giving the other half of (5.1).

(4) When $Q$ is a projection, $\alpha_{s}^{\psi}(Q)$ is the projection onto $\overline{\operatorname{span}} \psi_{s}\left(X_{s}\right) Q \mathcal{H}$, and the result follows from [13, Proposition 1.6(2)].

(5) See [13, Proposition 1.8(2)].

(6) $\rho_{s t}^{\psi}\left(\Theta_{z, w} \otimes_{A} 1\right)=\rho_{s t}^{\psi}(1) \rho_{s}^{\psi}\left(\Theta_{z, w}\right)=\alpha_{s t}^{\psi}(1) \psi(z) \psi(w)^{*}=\psi(z) \alpha_{t}^{\psi}(1) \psi(w)^{*}$. 
Proposition 5.6. Suppose $(G, P)$ is a quasi-lattice ordered group and $X$ is a product system over $P$ of essential Hilbert $A-A$ bimodules. A Toeplitz representation $\psi: X \rightarrow B(\mathcal{H})$ is Nica covariant if and only if

$$
\rho_{s}^{\psi}(S) \rho_{t}^{\psi}(T)= \begin{cases}\rho_{s \vee t}^{\psi}\left(\left(S \otimes_{A} 1\right)\left(T \otimes_{A} 1\right)\right) & \text { if } s \vee t<\infty \\ 0 & \text { otherwise }\end{cases}
$$

holds whenever $S \in \mathcal{K}\left(X_{s}\right)$ and $T \in \mathcal{K}\left(X_{t}\right)$.

Proof [10, Proposition 1.4]. If $\psi$ is Nica covariant, then

$$
\begin{aligned}
\rho_{s}^{\psi}(S) \rho_{t}^{\psi}(T) & =\rho_{s}^{\psi}(S) \rho_{s}^{\psi}(1) \rho_{t}^{\psi}(1) \rho_{t}^{\psi}(T) \\
& =\rho_{s}^{\psi}(S) \rho_{s \vee t}^{\psi}(1) \rho_{t}^{\psi}(T)=\rho_{s \vee t}^{\psi}\left(\left(S \otimes_{A} 1\right)\left(T \otimes_{A} 1\right)\right),
\end{aligned}
$$

where the last equality uses Lemma 5.5(5). Conversely, suppose (5.2) holds for all compact $S$ and $T$. If $S \rightarrow 1$ strictly, then

$$
\rho_{s \vee t}^{\psi}\left(\left(S \otimes_{A} 1\right)\right)=\rho_{s}^{\psi}(S) \rho_{s \vee t}^{\psi}(1) \rightarrow \rho_{s}^{\psi}(1) \rho_{s \vee t}^{\psi}(1)=\rho_{s \vee t}^{\psi}(1),
$$

where the convergence is in the strong operator topology. Hence

$$
\rho_{s}^{\psi}(1) \rho_{t}^{\psi}(T)= \begin{cases}\rho_{s \vee t}^{\psi}\left(T \otimes_{A} 1\right) & \text { if } s \vee t<\infty \\ 0 & \text { otherwise }\end{cases}
$$

for every $T \in \mathcal{K}\left(X_{t}\right)$. Letting $T \rightarrow 1$ strictly shows that $\psi$ is Nica covariant.

When each product $\left(S \otimes_{A} 1\right)\left(T \otimes_{A} 1\right)$ is compact, the previous Proposition allows us to give a $C^{*}$-algebraic characterization of Nica covariance:

Definition 5.7. Suppose $(G, P)$ is a quasi-lattice ordered group and $X$ is a product system over $P$ of essential Hilbert $A-A$ bimodules. We say that $X$ is compactly aligned if whenever $s, t \in P$ have a common upper bound and $S$ and $T$ are compact operators on $X_{s}$ and $X_{t}$, respectively, $\left(S \otimes_{A} 1\right)\left(T \otimes_{A} 1\right)$ is a compact operator on $X_{s \vee t}$. If $X$ is compactly aligned and $\psi$ is a Toeplitz representation of $X$ in a $C^{*}$-algebra $B$, we say that $\psi$ is Nica covariant if

$$
\psi^{(s)}(S) \psi^{(t)}(T)= \begin{cases}\psi^{(s \vee t)}\left(\left(S \otimes_{A} 1\right)\left(T \otimes_{A} 1\right)\right) & \text { if } s \vee t<\infty \\ 0 & \text { otherwise }\end{cases}
$$

whenever $s, t \in P, S \in \mathcal{K}\left(X_{s}\right)$ and $T \in \mathcal{K}\left(X_{t}\right)$.

Proposition 5.8. If $(G, P)$ is a total order, or if the left action of $A$ on each fiber $X_{s}$ is by compact operators, then $X$ is compactly aligned.

Proof. Suppose $s, t \in P, s \vee t<\infty, S \in \mathcal{K}\left(X_{s}\right)$, and $T \in \mathcal{K}\left(X_{t}\right)$. If $(G, P)$ is a total order then either $S \otimes_{A} 1=S$ or $T \otimes_{A} 1=T$; either way $\left(S \otimes_{A} 1\right)\left(T \otimes_{A} 1\right)$ is compact. If the left action of $A$ on each fiber $X_{s}$ is by compact operators, then by [22, Corollary 3.7], both $S \otimes_{A} 1$ and $T \otimes_{A} 1$ are compact. 
Proposition 5.9. Suppose $X$ is compactly aligned. Let $B$ and $C$ be $C^{*}$-algebras, let $\psi: X \rightarrow B$ be a Nica-covariant Toeplitz representation, and let $\sigma: B \rightarrow C$ be a homomorphism. Then $\sigma \circ \psi$ is Nica covariant.

Proof. By checking on an operator $\Theta_{x, y} \in \mathcal{K}\left(X_{s}\right)$, one verifies that $(\sigma \circ$ $\psi)^{(s)}=\sigma \circ \psi^{(s)}$, and the result follows easily from this.

Proposition 5.10. Suppose $X$ is a compactly-aligned product system, $\psi$ is a Nica-covariant Toeplitz representation of $X, s, t \in P, y \in X_{s}$, and $z \in X_{t}$. If $s \vee t=\infty$, then $\psi(y)^{*} \psi(z)=0$; otherwise

$$
\psi(y)^{*} \psi(z) \in \overline{\operatorname{span}}\left\{\psi(f) \psi(g)^{*}: f \in X_{s^{-1}(s \vee t)}, g \in X_{t^{-1}(s \vee t)}\right\} .
$$

Proof. Express $y=S y^{\prime}$ with $S \in \mathcal{K}\left(X_{s}\right)$ and $y^{\prime} \in X_{s}$; similarly, express $z=T z^{\prime}$ with $T \in \mathcal{K}\left(X_{t}\right)$ and $z^{\prime} \in X_{t}$. Since $\psi$ is Nica covariant,

$$
\psi(y)^{*} \psi(z)=\psi\left(y^{\prime}\right)^{*} \rho_{s}^{\psi}\left(S^{*}\right) \rho_{t}^{\psi}(T) \psi\left(z^{\prime}\right)
$$

is zero if $s \vee t=\infty$, and otherwise

$$
\psi(y)^{*} \psi(z)=\psi\left(y^{\prime}\right)^{*} \rho_{s \vee t}^{\psi}(K) \psi\left(z^{\prime}\right),
$$

where $K=\left(S^{*} \otimes_{A} 1\right)\left(T \otimes_{A} 1\right) \in \mathcal{K}\left(X_{s \vee t}\right)$. Since $K$ is compact it can be approximated in norm by a finite sum of operators $\Theta_{u, v}$ with $u, v \in X_{s \vee t}$, and hence $\rho_{s \vee t}^{\psi}(K)$ can be approximated by finite sums of the form $\psi(u) \psi(v)^{*}$. But any such $u$ can be approximated by finite sums of products $u_{1} f^{\prime}$ with $u_{1} \in X_{s}$ and $f^{\prime} \in X_{s^{-1}(s \vee t)}$; similarly, any such $v$ can be approximated by finite sums of products $v_{1} g^{\prime}$ with $v_{1} \in X_{t}$ and $g^{\prime} \in X_{t^{-1}(s \vee t)}$. Hence $\psi\left(y^{\prime}\right)^{*} \rho_{s \vee t}^{\psi}(K) \psi\left(z^{\prime}\right)$ can be approximated in norm by finite sums of operators of the form

$$
\psi\left(y^{\prime}\right)^{*} \psi\left(u_{1}\right) \psi\left(f^{\prime}\right) \psi\left(g^{\prime}\right)^{*} \psi\left(v_{1}\right)^{*} \psi\left(z^{\prime}\right)=\psi\left(\left\langle y^{\prime}, u_{1}\right\rangle_{A} f^{\prime}\right) \psi\left(\left\langle z^{\prime}, v_{1}\right\rangle_{A} g^{\prime}\right)^{*} .
$$

The following Lemma is useful when working with Nica-covariant Toeplitz representations.

Lemma 5.11. Suppose $(G, P)$ is a quasi-lattice ordered group, $X$ is a product system over $P$ of essential Hilbert $A-A$ bimodules, $\psi$ is a Toeplitz representation of $X$ on $\mathcal{H}, x \in X$, and $s \in P$.

(1) If $p(x) \leq s$, then $\alpha_{s}^{\psi}(S) \psi(x)=\psi(x) \alpha_{p(x)^{-1} s}^{\psi}(S)$ for all $S \in \psi_{e}(A)^{\prime}$.

(2) If $\psi$ is Nica covariant, then

$$
\alpha_{s}^{\psi}(1) \psi(x)= \begin{cases}\psi(x) \alpha_{p(x)^{-1}(p(x) \vee s)}^{\psi}(1) & \text { if } p(x) \vee s<\infty, \\ 0 & \text { otherwise. }\end{cases}
$$

Proof. The proof is formally identical to that of [12, Lemma 3.6]. 


\section{The system $\left(B_{P}, P, \tau, X\right)$.}

For each $t \in P$, let $1_{t} \in \ell^{\infty}(P)$ be the characteristic function of $t P$. Since the product $1_{s} 1_{t}$ is either $1_{s \vee t}$ or $0, B_{P}:=\overline{\operatorname{span}}\left\{1_{t}: t \in P\right\}$ is a $C^{*}$-subalgebra of $\ell^{\infty}(P)$. Left translation on $\ell^{\infty}(P)$ restricts to an action $\tau$ of $P$ on $B_{P}$, determined by $\tau_{s}\left(1_{t}\right)=1_{s t}$ for $s, t \in P$.

Proposition 6.1. Suppose $(G, P)$ is a quasi-lattice ordered group and $X$ is a product system over $P$ of essential Hilbert $A-A$ bimodules.

(1) If $(L, \psi)$ is a covariant representation of $\left(B_{P}, P, \tau, X\right)$, then $\psi$ is a nondegenerate Nica-covariant Toeplitz representation of $X$ and $L\left(1_{s}\right)=$ $\alpha_{s}^{\psi}(1)$.

(2) If $\psi$ is a nondegenerate Nica-covariant Toeplitz representation of $X$ on a Hilbert space $\mathcal{H}$, then there is a representation $L^{\psi}: B_{P} \rightarrow B(\mathcal{H})$ such that $L^{\psi}\left(1_{s}\right)=\alpha_{s}^{\psi}(1)$; moreover, $\left(L^{\psi}, \psi\right)$ is then a covariant representation of $\left(B_{P}, P, \tau, X\right)$.

Proof. The proof is formally identical to that of [12, Proposition 4.1], except that in (2) one must also note that $L^{\psi}\left(B_{P}\right) \subseteq \psi_{e}(A)^{\prime}$ since $L^{\psi}\left(1_{s}\right)=\alpha_{s}^{\psi}(1) \in$ $\psi_{e}(A)^{\prime}$ and $\left\{1_{s}: s \in P\right\}$ generates $B_{P}$.

Corollary 6.2. The system $\left(B_{P}, P, \tau, X\right)$ has a covariant representation.

Proof. Let $\pi$ be a nondegenerate representation of $A$ on a Hilbert space $\mathcal{H}$, and let $l: X \rightarrow \mathcal{L}(F(X))$ be the Fock representation of $X$. By Lemma 5.3, $\Psi:=F(X)-\operatorname{Ind}_{A}^{\mathcal{L}(F(X))} \pi \circ l$ is a Nica-covariant Toeplitz representation of $X$. Since $\pi$ is nondegenerate, so is $F(X)-\operatorname{Ind}_{A}^{\mathcal{L}(F(X))} \pi$; since $l$ is nondegenerate, $\Psi$ is as well. The previous Proposition thus gives a covariant representation $\left(L^{\Psi}, \Psi\right)$ of $\left(B_{P}, P, \tau, X\right)$.

Let $i_{X}$ and $i_{B_{P}}$ be the canonical maps of $X$ and $B_{P}$ into $M\left(B_{P} \rtimes_{\tau, X} P\right)$. Since $B_{P}$ is unital, $i_{X}(x)=i_{B_{P}}(1) i_{X}(x) \in B_{P} \rtimes_{\tau, X} P$ for each $x \in X$. We write $\mathcal{T}_{\text {cov }}(X)$ for the $C^{*}$-subalgebra of $B_{P} \rtimes_{\tau, X} P$ generated by $i_{X}(X)$; the following Theorem justifies this notation.

Theorem 6.3. $\left(\mathcal{I}_{\operatorname{cov}}(X), i_{X}\right)$ is universal for Nica-covariant Toeplitz representations of $X$, in the sense that:

(a) There is a faithful representation $\theta$ of $\mathcal{T}_{\text {cov }}(X)$ on Hilbert space such that $\theta \circ i_{X}$ is a Nica-covariant Toeplitz representation of $X$; and

(b) for every Nica-covariant Toeplitz representation $\psi$ of $X$, there is a representation $\psi_{*}$ of $\mathcal{T}_{\text {cov }}(X)$ such that $\psi=\psi_{*} \circ i_{X}$.

Up to canonical isomorphism, $\left(\mathcal{T}_{\mathrm{cov}}(X), i_{X}\right)$ is the unique pair with this property. If $X$ is compactly aligned, then $i_{X}$ is Nica covariant,

$$
\mathcal{T}_{\text {cov }}(X)=\overline{\operatorname{span}}\left\{i_{X}(x) i_{X}(y)^{*}: x, y \in X\right\},
$$


and

$$
B_{P} \rtimes_{\tau, X} P=\overline{\operatorname{span}}\left\{i_{X}(x) i_{B_{P}}\left(1_{s}\right) i_{X}(y)^{*}: x, y \in X, s \in P\right\} .
$$

If the left action of $A$ on each fiber $X_{s}$ is by compact operators, then $\mathcal{T}_{\text {cov }}(X)$ is all of $B_{P} \rtimes_{\tau, X} P$; if in addition every $s, t \in P$ have a common upper bound, then the Cuntz-Pimsner algebra $\mathcal{O}_{X}$ is a quotient of $\mathcal{T}_{\text {cov }}(X)$.

Proof of Theorem 6.3. Let $\sigma$ be a faithful representation of $B_{P} \rtimes_{\tau, X} P$ on a Hilbert space $\mathcal{H}$ such that $\left(\bar{\sigma} \circ i_{B_{P}}, \sigma \circ i_{X}\right)$ is a covariant representation of $\left(B_{P}, P, \tau, X\right)$. By Proposition 6.1(1), $\sigma \circ i_{X}$ is a Nica-covariant Toeplitz representation of $X$, so we can take $\theta$ to be the restriction of $\sigma$ to $\mathcal{T}_{\text {cov }}(X)$. Suppose $\psi$ is a (nondegenerate) Nica-covariant Toeplitz representation of $X$. Proposition 6.1(2) gives us a covariant representation $\left(L^{\psi}, \psi\right)$ of $\left(B_{P}, P, \tau, X\right)$, and hence a representation $L^{\psi} \times \psi$ of $B_{P} \rtimes_{\tau, X} P$ such that $\left(L^{\psi} \times \psi\right) \circ i_{X}=\psi$. Restricting $L^{\psi} \times \psi$ to $\mathcal{T}_{\text {cov }}(X)$ gives the required representation $\psi_{*}$. Uniqueness of $\left(\mathcal{T}_{\text {cov }}(X), i_{X}\right)$ follows by the usual argument.

Suppose $X$ is compactly aligned. Since $i_{X}$ is the composition of the Nicacovariant Toeplitz representation $\sigma \circ i_{X}$ and the homomorphism $\sigma^{-1}$ (restricted to $\left.\sigma\left(\mathcal{T}_{\text {cov }}(X)\right)\right), i_{X}$ is Nica covariant by Proposition 5.9. Let $w \in X$, and express $w=z \cdot a$ for some $z \in X$ and $a \in A$. Then $i_{X}(w)=i_{X}(z) i_{X}\left(a^{*}\right)^{*}$, so $\mathcal{A}:=\overline{\operatorname{span}}\left\{i_{X}(x) i_{X}(y)^{*}: x, y \in X\right\}$ contains $i_{X}(X)$. Obviously $\mathcal{A}$ is a closed self-adjoint subspace of $\mathcal{T}_{\text {cov }}(X)$, and since $X$ is compactly aligned, Proposition 5.10 shows that $\mathcal{A}$ is closed under multiplication. This gives (6.1).

Now let $\mathcal{B}:=\overline{\operatorname{span}}\left\{i_{X}(x) i_{B_{P}}\left(1_{s}\right) i_{X}(y)^{*}: x, y \in X, s \in P\right\}$. Using Lemma 5.11 with $\psi:=\sigma \circ i_{X}$, and then applying $\sigma^{-1}$, gives

$$
i_{X}(x) i_{B_{P}}\left(1_{s}\right)=i_{B_{P}}\left(1_{p(x) s}\right) i_{X}(x)
$$

and

$$
i_{B_{P}}\left(1_{s}\right) i_{X}(x)= \begin{cases}i_{X}(x) i_{B_{P}}\left(1_{p(x)^{-1}(p(x) \vee s)}\right) & \text { if } p(x) \vee s<\infty \\ 0 & \text { otherwise. }\end{cases}
$$

Equation (6.3) shows that

$$
i_{X}(x) i_{B_{P}}\left(1_{s}\right) i_{X}(y)^{*}=i_{B_{P}}\left(1_{p(x) s}\right) i_{X}(x)\left(i_{B_{P}}\left(1_{p(y) s}\right) i_{X}(y)\right)^{*} \in B_{P} \rtimes_{\tau, X} P,
$$

so $\mathcal{B} \subseteq B_{P} \rtimes_{\tau, X} P$. Since $B_{P}$ is generated by $\left\{1_{s}: s \in P\right\}$, elements of the form $i_{B_{P}}\left(1_{s}\right) i_{X}(w)$ generate $B_{P} \rtimes_{\tau, X} P$ as a $C^{*}$-algebra; with $w=z \cdot a$ as 
above, (6.4) shows that

$$
\begin{aligned}
i_{B_{P}}\left(1_{s}\right) i_{X}(w) & =i_{B_{P}}\left(1_{s}\right) i_{X}(z) i_{X}\left(a^{*}\right)^{*} \\
& = \begin{cases}i_{X}(z) i_{B_{P}}\left(1_{p(z)^{-1}(p(z) \vee s)}\right) i_{X}\left(a^{*}\right)^{*} & \text { if } p(z) \vee s<\infty \\
0 & \text { otherwise }\end{cases} \\
& \in \mathcal{B} .
\end{aligned}
$$

Hence to establish (6.2), it remains only to show that $\mathcal{B}$ is closed under multiplication. But Proposition 5.10 shows that the product

$$
i_{X}(x) i_{B_{P}}\left(1_{s}\right) i_{X}(y)^{*} i_{X}(z) i_{B_{P}}\left(1_{t}\right) i_{X}(w)^{*}
$$

of two typical generators of $\mathcal{B}$ is contained in the closed linear span of elements of the form

$$
i_{X}(x) i_{B_{P}}\left(1_{s}\right) i_{X}(f) i_{X}(g)^{*} i_{B_{P}}\left(1_{t}\right) i_{X}(w)^{*},
$$

which by (6.4) simplifies to

$$
i_{X}(x f) i_{B_{P}}\left(1_{p(f)^{-1}(p(f) \vee s) \vee p(g)^{-1}(p(g) \vee t)}\right) i_{X}(w g)^{*} \in \mathcal{B} .
$$

Suppose the left action of $A$ on each $X_{s}$ is by compact operators; that is, $\phi_{s}(A) \subseteq \mathcal{K}\left(X_{s}\right)$ for all $s \in P$. Let $x \in X$ and $s \in P$. Since $X_{p(x)}$ is essential, we can express $x=\phi_{p(x)}(a) z$ for some $a \in A$ and $z \in X_{p(x)}$. With $\psi:=\sigma \circ i_{X}$, we then have

$$
\begin{aligned}
\sigma\left(i_{B_{P}}\left(1_{s}\right) i_{X}(x)\right) & =L^{\psi}\left(1_{s}\right) \psi(x)=\rho_{s}^{\psi}(1) \psi_{e}(a) \psi(z) \\
& =\psi^{(s)}\left(\phi_{s}(a)\right) \psi(z) \\
& =\sigma\left(i_{X}^{(s)}\left(\phi_{s}(a)\right) i_{X}(z)\right)
\end{aligned}
$$

so $i_{B_{P}}\left(1_{s}\right) i_{X}(x)=i_{X}^{(s)}\left(\phi_{s}(a)\right) i_{X}(z) \in \mathcal{T}_{\text {cov }}(X)$. Since elements of the form $i_{B_{P}}\left(1_{s}\right) i_{X}(x)$ generate $B_{P} \rtimes_{\tau, X} P$, this gives $B_{P} \rtimes_{\tau, X} P=\mathcal{T}_{\text {cov }}(X)$.

If in addition every $s, t \in P$ have a common upper bound, then by Proposition 5.4 the universal map $j_{X}: X \rightarrow \mathcal{O}_{X}$ is Nica covariant; the integrated form $\left(j_{X}\right)_{*}: \mathcal{T}_{\text {cov }}(X) \rightarrow \mathcal{O}_{X}$ is surjective since it maps generators to generators.

\section{Faithful representations.}

Our strategy for characterizing faithful representations of $B_{P} \rtimes_{\tau, X} P$ follows $\left[12\right.$, Section 5]. First we use the dual coaction $\delta$ of $G$ on $B_{P} \rtimes_{\tau, X} P$ and the canonical trace $\rho$ on $C^{*}(G)$ to define a positive linear map $E_{\delta}:=($ id $\otimes \rho) \circ \delta$ of norm one of $B_{P} \rtimes_{\tau, X} P$ onto the fixed-point algebra $\left(B_{P} \rtimes_{\tau, X} P\right)^{\delta}$. When $X$ is compactly aligned, $\left(B_{P}, P, \tau, X\right)$ satisfies the spanning condition (6.2), 
and $E_{\delta}$ is determined by

$$
E_{\delta}\left(i_{X}(x) i_{B_{P}}\left(1_{s}\right) i_{X}(y)^{*}\right)= \begin{cases}i_{X}(x) i_{B_{P}}\left(1_{s}\right) i_{X}(y)^{*} & \text { if } p(x)=p(y) \\ 0 & \text { otherwise. }\end{cases}
$$

Definition 7.1. The system $\left(B_{P}, P, \tau, X\right)$ is amenable if $E_{\delta}$ is faithful on positive elements.

The argument of $[\mathbf{1 7}$, Lemma 6.5] shows that if $G$ is an amenable group, then the system $\left(B_{P}, P, \tau, X\right)$ is amenable. In Corollary 8.2 we will show that $\left(B_{P}, P, \tau, X\right)$ is also amenable when $X$ is compactly aligned and $G$ is a free product $*\left(G^{\lambda}, P^{\lambda}\right)$ with each $G^{\lambda}$ an amenable group.

Theorem 7.2. Suppose $(G, P)$ is a quasi-lattice ordered group and $X$ is a compactly-aligned product system over $P$ of essential Hilbert $A-A$ bimodules such that the system $\left(B_{P}, P, \tau, X\right)$ is amenable. Let $\psi$ be a Nica-covariant Toeplitz representation of $X$ on a Hilbert space $\mathcal{H}$. Then $L^{\psi} \times \psi$ is a faithful representation of $B_{P} \rtimes_{\tau, X} P$ if and only if

(7.2) for every $n \geq 1$ and $s_{1}, \ldots, s_{n} \in P \backslash\{e\}$, the subrepresentation

$$
a \in A \mapsto \psi_{e}(a) \prod_{k=1}^{n}\left(1-L^{\psi}\left(1_{s_{k}}\right)\right) \text { of } \psi_{e} \text { is faithful. }
$$

Proof of necessity of (7.2). Let $\pi: A \rightarrow B(\mathcal{H})$ be a faithful nondegenerate representation of $A$ on a Hilbert space $\mathcal{H}$, let $l: X \rightarrow \mathcal{L}(F(X))$ be the Fock representation of $X$, and let $\Psi:=F(X)-\operatorname{Ind}_{A} \mathcal{L}(F(X)) \pi \circ l$; by Lemma 5.3, $\Psi$ is a Nica-covariant Toeplitz representation of $X$ on $F(X) \otimes_{A} \mathcal{H}$. We claim that

$$
a \in A \mapsto \Psi_{e}(a) \prod_{k=1}^{n}\left(1-L^{\Psi}\left(1_{s_{k}}\right)\right)
$$

is faithful. Since $L^{\Psi}\left(1_{s_{k}}\right)=\alpha_{s_{k}}^{\Psi}(1)$ is the orthogonal projection of $F(X) \otimes_{A}$ $\mathcal{H}$ onto $\bigoplus_{t \in s_{k} P} X_{t} \otimes_{A} \mathcal{H}$ (see the proof of Lemma 5.3), each projection $1-L^{\Psi}\left(1_{s_{k}}\right)$ dominates the projection $Q_{e}$ onto the $\Psi_{e}$-invariant subspace $X_{e} \otimes_{A} \mathcal{H}$. To establish the claim it thus suffices to show that the subrepresentation $Q_{e} \Psi_{e}$ of $\Psi_{e}$ is faithful. But $\Psi_{e}=F(X)-\operatorname{Ind}_{A}^{A} \pi$ decomposes as $\bigoplus_{t \in P} X_{t}-\operatorname{Ind}_{A}^{A} \pi$, so $Q_{e} \Psi_{e}=A-\operatorname{Ind}_{A}^{A} \pi$ is unitarily equivalent to $\pi$, and hence faithful.

Now suppose that $L^{\psi} \times \psi$ is faithful and $a \in A$. Let

$$
T:=i_{B_{P}}\left(\prod_{k=1}^{n}\left(1-1_{s_{k}}\right)\right) i_{X}(a) \in B_{P} \rtimes_{\tau, X} P .
$$


Then

$$
\begin{aligned}
\|a\| & =\left\|\Psi_{e}(a) \prod_{k=1}^{n}\left(1-L^{\Psi}\left(1_{s_{k}}\right)\right)\right\|=\left\|L^{\Psi} \times \Psi(T)\right\| \leq\|T\| \\
& =\left\|L^{\psi} \times \psi(T)\right\|=\left\|\psi_{e}(a) \prod_{k=1}^{n}\left(1-L^{\psi}\left(1_{s_{k}}\right)\right)\right\| \leq\|a\|,
\end{aligned}
$$

giving (7.2).

Our proof that (7.2) implies faithfulness of $L^{\psi} \times \psi$ is based on the argument of [12, Section 6]: In Proposition 7.5(1) we prove that $L^{\psi} \times \psi$ is faithful on $\left(B_{P} \rtimes_{\tau, X} P\right)^{\delta}$, and in Proposition 7.5(2) we construct a spatial version $E_{\psi}$ of $E_{\delta}$ such that $\left(L^{\psi} \times \psi\right) \circ E_{\delta}=E_{\psi} \circ\left(L^{\psi} \times \psi\right)$. Faithfulness of $L^{\psi} \times \psi$ then follows easily: If $L^{\psi} \times \psi(b)=0$, then

$$
0=E_{\psi} \circ\left(L^{\psi} \times \psi\right)\left(b^{*} b\right)=\left(L^{\psi} \times \psi\right) \circ E_{\delta}\left(b^{*} b\right),
$$

so by Proposition $7.5(1), E_{\delta}\left(b^{*} b\right)=0$. The amenability hypothesis then forces $b^{*} b=0$, and hence $b=0$.

We begin by reviewing some notation and results from [17, Remark 1.5] and [12, Remark 5.2]. Let $F$ be a finite subset of $P$. A subset $C$ of $F$ is an initial segment of $F$ if $c:=\sigma C$ is finite and $C=\{t \in F: t \leq c\}$. (Recall that $\sigma C$ is the least upper bound of $C$; we use the convention that $\sigma \emptyset=e$.) For each such $C$ there is a nonzero projection $Q_{C}$ in $B_{P}$ defined by

$$
Q_{C}:=1_{c} \prod_{\{t \in F: c<t \vee c<\infty\}}\left(1-1_{t}\right),
$$

and as $C$ ranges over the initial segements of $F$, these projections form a decomposition of the identity in $B_{P}$.

Lemma 7.3. Suppose $(G, P)$ is a quasi-lattice ordered group, $X$ is a product system over $P$ of essential Hilbert $A-A$ bimodules, $\psi$ is a Nica-covariant Toeplitz representation of $X$ on $\mathcal{H}, F$ is a finite subset of $P, C$ is an initial segment of $F, x, y \in X$ and $s \in P$. Let $c=\sigma C$, so that $C=\{t \in F: t \leq c\}$.

(1) If $p(x)=p(y)$, then the operator $\psi(x) L^{\psi}\left(1_{s}\right) \psi(y)^{*}$ is in the commutant of $L^{\psi}\left(B_{P}\right)$. In particular, it commutes with $L^{\psi}\left(Q_{C}\right)$.

(2) If $p(x) s, p(y) s \in F$, then

$$
\begin{aligned}
L^{\psi}\left(Q_{C}\right) \psi(x) L^{\psi}\left(1_{s}\right) \psi(y)^{*} L^{\psi}\left(Q_{C}\right) \\
\quad=\left\{\begin{array}{c}
L^{\psi}\left(Q_{C}\right) \psi(x) L^{\psi}\left(1_{p(x)-1} c L^{\psi}\left(1_{p(y)^{-1} c}\right) \psi(y)^{*} L^{\psi}\left(Q_{C}\right)\right. \\
0 \quad \text { otherwise. } \quad \text { if } p(x) s \leq c \text { and } p(y) s \leq c
\end{array}\right.
\end{aligned}
$$

Proof. The proof, based on Lemma 5.11, is identical in form to the proof of [12, Lemma 5.3]. 
Lemma 7.4. Suppose $(G, P)$ is a quasi-lattice ordered group, $X$ is a product system over $P$ of essential Hilbert $A-A$ bimodules, and $\psi$ is a Nica-covariant Toeplitz representation of $X$ which satisfies (7.2). Suppose further that $F$ is a finite subset of $P$ and $Z$ is a finite sum $\sum \psi\left(x_{k}\right) L^{\psi}\left(1_{s_{k}}\right) \psi\left(y_{k}\right)^{*}$ such that $p\left(x_{k}\right) s_{k}=p\left(y_{k}\right) s_{k} \in F$ for each $k$. Then

$$
\|Z\|=\max \left\{\left\|T_{C}\right\|: C \text { is an initial segment of } F\right\},
$$

where $T_{C}$ is the adjointable operator on $X_{\sigma C}$ defined by

$$
T_{C}:=\sum_{p\left(x_{k}\right) s_{k} \leq \sigma C} \Theta_{x_{k}, y_{k}} \otimes_{A} 1^{p\left(x_{k}\right)^{-1} \sigma C} .
$$

Proof. Since $\left\{Q_{C}: C\right.$ is an initial segment of $\left.F\right\}$ is a decomposition of the identity in $B_{P}$, and since $L^{\psi}$ is a unital representation of $B_{P}$, the projections $L^{\psi}\left(Q_{C}\right)$ decompose the identity operator. By Lemma 7.3(1), $Z$ commutes with each $L^{\psi}\left(Q_{C}\right)$, and thus

$$
\|Z\|=\max \left\{\left\|L^{\psi}\left(Q_{C}\right) Z\right\|: C \text { is an initial segment of } F\right\} .
$$

Fix an initial segment $C$, and let $c:=\sigma C$. By Lemma 7.3(2) and Lemma 5.5(6),

$$
\begin{aligned}
L^{\psi}\left(Q_{C}\right) Z & =L^{\psi}\left(Q_{C}\right) \sum \psi\left(x_{k}\right) L^{\psi}\left(1_{s_{k}}\right) \psi\left(y_{k}\right)^{*} \\
& =L^{\psi}\left(Q_{C}\right) \sum_{p\left(x_{k}\right) s_{k} \leq c} \psi\left(x_{k}\right) L^{\psi}\left(1_{p\left(x_{k}\right)^{-1}}\right) \psi\left(y_{k}\right)^{*} \\
& =L^{\psi}\left(Q_{C}\right) \sum_{p\left(x_{k}\right) s_{k} \leq c} \rho_{c}^{\psi}\left(\Theta_{x_{k}, y_{k}} \otimes_{A} 1\right) \\
& =L^{\psi}\left(Q_{C}\right) \rho_{c}^{\psi}\left(T_{C}\right),
\end{aligned}
$$

so it suffices to show that

$$
\left\|L^{\psi}\left(Q_{C}\right) \rho_{c}^{\psi}\left(T_{C}\right)\right\|=\left\|T_{C}\right\| .
$$

Let

$$
R_{C}:=\prod_{\{t \in F: c<t \vee c<\infty\}}\left(1-1_{c^{-1}(t \vee c)}\right) \in B_{P} .
$$

Since $\psi$ satisfies (7.2),

$$
a \mapsto \psi_{e}(a) \prod_{\{t \in F: c<t \vee c<\infty\}}\left(1-L^{\psi}\left(1_{c^{-1}(t \vee c)}\right)\right)=\psi_{e}(a) L^{\psi}\left(R_{C}\right)
$$

is a faithful representation of $A$. By Lemma 5.5(4), the representation $T \in \mathcal{L}\left(X_{c}\right) \mapsto \alpha_{c}^{\psi}\left(L^{\psi}\left(R_{C}\right)\right) \rho_{c}^{\psi}(T)$ is thus also faithful. But $\alpha_{c}^{\psi}\left(L^{\psi}\left(R_{C}\right)\right)=$ $L^{\psi}\left(\tau_{c}\left(R_{C}\right)\right)=L^{\psi}\left(Q_{C}\right)$, and hence (7.5) is satisfied. 
Proposition 7.5. Suppose $(G, P)$ is a quasi-lattice ordered group, $X$ is a compactly-aligned product system over $P$ of essential Hilbert $A-A$ bimodules, and $\psi$ is a Nica-covariant Toeplitz representation of $X$ which satisfies (7.2).

(1) $L^{\psi} \times \psi$ is isometric on $\left(B_{P} \rtimes_{\tau, X} P\right)^{\delta}$.

(2) There is a linear map $E_{\psi}$ of norm one of $L^{\psi} \times \psi\left(B_{P} \rtimes_{\tau, X} P\right)$ onto $L^{\psi} \times \psi\left(\left(B_{P} \rtimes_{\tau, X} P\right)^{\delta}\right)$ such that $E_{\psi} \circ\left(L^{\psi} \times \psi\right)=\left(L^{\psi} \times \psi\right) \circ E_{\delta}$.

Proof. (1) Since $X$ is compactly aligned, the spanning condition (6.2) holds. Since $E_{\delta}$ is continuous and maps onto $\left(B_{P} \rtimes_{\tau, X} P\right)^{\delta}$, we deduce that finite sums

$$
z:=\sum i_{X}\left(x_{k}\right) i_{B_{P}}\left(1_{s_{k}}\right) i_{X}\left(y_{k}\right)^{*}
$$

in which $p\left(x_{k}\right)=p\left(y_{k}\right)$ for all $k$ are dense in $\left(B_{P} \rtimes_{\tau, X} P\right)^{\delta}$. It therefore suffices to fix such a $z$ and show that $\left\|L^{\psi} \times \psi(z)\right\|=\|z\|$.

Let $\sigma$ be a faithful nondegenerate representation of $B_{P} \rtimes_{\tau, X} P$ such that $\left(\bar{\sigma} \circ i_{B_{P}}, \sigma \circ i_{X}\right)$ is a covariant representation of $\left(B_{P}, P, \tau, X\right)$. By Proposition $6.1, i:=\sigma \circ i_{X}$ is a covariant representation of $X$ and $\bar{\sigma} \circ i_{B_{P}}=L^{i}$. Since $L^{i} \times i=\sigma$ is faithful, $i$ satisfies (7.2). Hence with $F:=\left\{p\left(x_{k}\right) s_{k}\right\}$, Lemma 7.4 gives

$$
\begin{aligned}
\left\|L^{\psi} \times \psi(z)\right\| & =\left\|\sum \psi\left(x_{k}\right) L^{\psi}\left(1_{s_{k}}\right) \psi\left(y_{k}\right)^{*}\right\| \\
& =\max \left\{\left\|T_{C}\right\|: C \text { is an initial segment of } F\right\} \\
& =\left\|\sum i\left(x_{k}\right) L^{i}\left(1_{s_{k}}\right) i\left(y_{k}\right)^{*}\right\|=\left\|L^{i} \times i(z)\right\|=\|z\| .
\end{aligned}
$$

(2) Since $X$ is compactly aligned, finite sums of the form

$$
w:=\sum i_{X}\left(x_{k}\right) i_{B_{P}}\left(1_{s_{k}}\right) i_{X}\left(y_{k}\right)^{*}
$$

are dense in $B_{P} \rtimes_{\tau, X} P$. We will show that $\left\|L^{\psi} \times \psi\left(E_{\delta}(w)\right)\right\| \leq\left\|L^{\psi} \times \psi(w)\right\|$; it follows that $E_{\psi}$ is well-defined on operators of the form $L^{\psi} \times \psi(w)$ and extends to the desired linear contraction.

Let $F:=\left\{p\left(x_{k}\right) s_{k}\right\} \cup\left\{p\left(y_{k}\right) s_{k}\right\}$, and let $Z:=L^{\psi} \times \psi\left(E_{\delta}(w)\right)$; by (7.1),

$$
Z=\sum_{p\left(x_{k}\right)=p\left(y_{k}\right)} \psi\left(x_{k}\right) L^{\psi}\left(1_{s_{k}}\right) \psi\left(y_{k}\right)^{*} .
$$

By Lemma 7.4, there is an initial segment $C$ of $F$ such that $\|Z\|=\left\|T_{C}\right\|$. Let $c:=\sigma C$. We will construct a projection $R \in B_{P}$ such that $a \in A \mapsto$ $\psi_{e}(a) L^{\psi}(R)$ is faithful, then define $Q:=L^{\psi}\left(\tau_{c}(R)\right)=\alpha_{c}^{\psi}\left(L^{\psi}(R)\right)$, and show that $Q\left(L^{\psi} \times \psi(w)\right) Q=Q \rho_{c}^{\psi}\left(T_{C}\right)$. This will complete the proof, since by Lemma 5.5(4) we then have

$$
\|Z\|=\left\|T_{C}\right\|=\left\|Q \rho_{c}^{\psi}\left(T_{C}\right)\right\|=\left\|Q\left(L^{\psi} \times \psi(w)\right) Q\right\| \leq\left\|L^{\psi} \times \psi(w)\right\| .
$$


For each $s, t \in C$ such that $s \neq t$ and $s^{-1} c \vee t^{-1} c<\infty$, define $d_{s, t} \in P$ as in [17, Lemma 3.2]:

$$
d_{s, t}= \begin{cases}\left(s^{-1} c\right)^{-1}\left(s^{-1} c \vee t^{-1} c\right) & \text { if } s^{-1} c<s^{-1} c \vee t^{-1} c \\ \left(t^{-1} c\right)^{-1}\left(s^{-1} c \vee t^{-1} c\right) & \text { otherwise, }\end{cases}
$$

noting in particular that $d_{s, t}$ is never the identity in $P$. Let $R_{C}$ be as in (7.6), and define

$$
R:=R_{C} \prod_{\substack{s \neq t \in C \\ s \neq c \\ s^{-1} \\ c \vee t^{-1} c<\infty}}\left(1-1_{d_{s, t}}\right) .
$$

By condition (7.2), $a \in A \mapsto L^{\psi}(R) \psi_{e}(a)$ is faithful. The proof that $Q\left(L^{\psi} \times\right.$ $\psi(w)) Q=Q \rho_{c}^{\psi}\left(T_{C}\right)$ is exactly as in [12, Proposition 5.5], so we omit it.

Proposition 7.6. Suppose $(G, P)$ is a quasi-lattice ordered group and $X$ is a compactly-aligned product system over $P$ of essential Hilbert $A-A$ bimodules. Let $\pi$ be a nondegenerate representation of $A$ on a Hilbert space $\mathcal{H}$, and let $\Psi$ be the representation $F(X)-\operatorname{Ind}_{A}{ }^{\mathcal{L}(F(X))} \pi \circ l$, where $l: X \rightarrow \mathcal{L}(F(X))$ is the Fock representation of $X$. There is a projection $E_{\Psi}$ of norm one of $L^{\Psi} \times \Psi\left(B_{P} \rtimes_{\tau, X} P\right)$ onto $L^{\Psi} \times \Psi\left(\left(B_{P} \rtimes_{\tau, X} P\right)^{\delta}\right)$ such that

$$
E_{\Psi} \circ\left(L^{\Psi} \times \Psi\right)=\left(L^{\Psi} \times \Psi\right) \circ E_{\delta} ;
$$

moreover, $E_{\Psi}$ is faithful on positive operators.

Proof. Denote by $Q_{t}$ the orthogonal projection of $F(X) \otimes_{A} \mathcal{H}$ onto $X_{t} \otimes_{A} \mathcal{H}$. Since the $Q_{t}$ 's are mutually orthogonal, the formula

$$
E_{\Psi}(T):=\sum_{t \in P} Q_{t} T Q_{t} \quad \text { for } T \in L^{\Psi} \times \Psi\left(B_{P} \rtimes_{\tau, X} P\right)
$$

defines a completely positive projection of norm one which is faithful on positive operators. We claim that

$$
E_{\Psi}\left(\Psi(x) L^{\Psi}\left(1_{s}\right) \Psi(y)^{*}\right)= \begin{cases}\Psi(x) L^{\Psi}\left(1_{s}\right) \Psi(y)^{*} & \text { if } p(x)=p(y) \\ 0 & \text { otherwise. }\end{cases}
$$

Since $X$ is compactly aligned the spanning condition (6.2) holds, and hence (7.7) follows from (7.8) and (7.1).

Suppose $x, y \in X$ and $s \in P$. For each $t \in P, \Psi(x) L^{\Psi}\left(1_{s}\right) \Psi(y)^{*}$ is zero on $X_{t} \otimes_{A} \mathcal{H}$ unless $p(y) s \leq t$, in which case $\Psi(x) L^{\Psi}\left(1_{s}\right) \Psi(y)^{*}$ maps $X_{t} \otimes_{A} \mathcal{H}$ into $X_{p(x) p(y)^{-1} t} \otimes_{A} \mathcal{H}$. Thus if $p(x) \neq p(y), Q_{t} \Psi(x) L^{\Psi}\left(1_{s}\right) \Psi(y)^{*} Q_{t}=0$ for every $t \in P$, and $E_{\Psi}\left(\Psi(x) L^{\Psi}\left(1_{s}\right) \Psi(y)^{*}\right)=0$. If on the other hand $p(x)=p(y)$, 
then $Q_{t} \Psi(x) L^{\Psi}\left(1_{s}\right) \Psi(y)^{*} Q_{t}=\Psi(x) L^{\Psi}\left(1_{s}\right) \Psi(y)^{*} Q_{t}$ for each $t \in P$, and thus

$$
\begin{aligned}
E_{\Psi}\left(\Psi(x) L^{\Psi}\left(1_{s}\right) \Psi(y)^{*}\right) & =\sum_{t \in P} Q_{t} \Psi(x) L^{\Psi}\left(1_{s}\right) \Psi(y)^{*} Q_{t} \\
& =\Psi(x) L^{\Psi}\left(1_{s}\right) \Psi(y)^{*} \sum_{t \in P} Q_{t}=\Psi(x) L^{\Psi}\left(1_{s}\right) \Psi(y)^{*} .
\end{aligned}
$$

Corollary 7.7. Suppose $\pi$ is faithful. Then the system $\left(B_{P}, P, \tau, X\right)$ is amenable if and only if the representation $L^{\Psi} \times \Psi$ of $B_{P} \rtimes_{\tau, X} P$ is faithful.

Proof. Suppose $L^{\Psi} \times \Psi$ is faithful. By Proposition 7.6, $\left(L^{\Psi} \times \Psi\right) \circ E_{\delta}=$ $E_{\Psi} \circ\left(L^{\Psi} \times \Psi\right)$ is faithful on positive elements, hence so is $E_{\delta}$; that is, $\left(B_{P}, P, \tau, X\right)$ is amenable. Since $\Psi$ satisfies (7.2) (see the proof of necessity of (7.2)), the converse follows from Theorem 7.2.

\section{Amenability.}

Theorem 8.1. Suppose $\theta:(G, P) \rightarrow(\mathcal{G}, \mathcal{P})$ is a homomorphism of quasilattice ordered groups such that, whenever $s \vee t<\infty$,

$$
\theta(s \vee t)=\theta(s) \vee \theta(t) \text { and } \theta(s)=\theta(t) \Longrightarrow s=t,
$$

and suppose that $\mathcal{G}$ is amenable. If $X$ is a compactly-aligned product system over $P$ of essential Hilbert $A-A$ bimodules, then the system $\left(B_{P}, P, \tau, X\right)$ is amenable.

Proof. Our proof is essentially that of [12, Theorem 6.1], suitably modified to handle Hilbert bimodules. The homomorphism $\theta: G \rightarrow \mathcal{G}$ induces a coaction $\delta_{\theta}=(\mathrm{id} \otimes \theta) \circ \delta$ of $\mathcal{G}$ on $B_{P} \rtimes_{\tau, X} P$, and hence a conditional expectation $E_{\delta_{\theta}}$ of $B_{P} \rtimes_{\tau, X} P$ onto the fixed-point algebra $\left(B_{P} \rtimes_{\tau, X} P\right)^{\delta_{\theta}}$, such that

$$
E_{\delta_{\theta}}\left(i_{X}(x) i_{B_{P}}\left(1_{s}\right) i_{X}(y)^{*}\right)= \begin{cases}i_{X}(x) i_{B_{P}}\left(1_{s}\right) i_{X}(y)^{*} & \text { if } \theta(p(x))=\theta(p(y)) \\ 0 & \text { otherwise. }\end{cases}
$$

Since $\mathcal{G}$ is amenable, $E_{\delta_{\theta}}$ is faithful on positive elements.

Let $l: X \rightarrow \mathcal{L}(F(X))$ be the Fock representation of $X$, let $\pi$ be a faithful nondegenerate representation of $A$ on a Hilbert space $\mathcal{H}$, and let $\Psi:=F(X)-\operatorname{Ind}_{A}^{\mathcal{L}(F(X))} \pi \circ l$. By Proposition 7.6, for every $b \in B_{P} \rtimes_{\tau, X} P$ we have

$$
\left(L^{\Psi} \times \Psi\right) \circ E_{\delta}(b)=E_{\Psi}\left(L^{\Psi} \times \Psi\left(E_{\delta_{\theta}}(b)\right)\right) .
$$

Since $E_{\delta_{\theta}}$ and $E_{\Psi}$ are faithful on positive elements, to show that $\left(B_{P}, P, \tau, X\right)$ is amenable it suffices to show that $L^{\Psi} \times \Psi$ is faithful on $\left(B_{P} \rtimes_{\tau, X} P\right)^{\delta_{\theta}}$.

Let $\sigma$ be a faithful representation of $B_{P} \rtimes_{\tau, X} P$ such that $\left(\bar{\sigma} \circ i_{B_{P}}, \sigma \circ i_{X}\right)$ is a covariant representation of $\left(B_{P}, P, \tau, X\right)$. By Proposition $6.1, i=\sigma \circ i_{X}$ is 
a covariant representation of $X$ and $\bar{\sigma} \circ i_{B_{P}}=L^{i}$. Observe that $i$ is isometric since, by Lemma 5.3,

$$
\begin{aligned}
\|x\|=\|\Psi(x)\| & =\left\|\left(L^{\Psi} \times \Psi\right) \circ i_{X}(x)\right\| \\
& \leq\left\|i_{X}(x)\right\|=\left\|\sigma \circ i_{X}(x)\right\|=\|i(x)\| \leq\|x\| .
\end{aligned}
$$

Let $\mathcal{F}$ be the set of all finite subsets $F$ of $\mathcal{P}$ which are closed under $\vee$ in the sense that $s \vee t \in F$ whenever $s, t \in F$ and $s \vee t<\infty$. Exactly as in the proof of [12, Theorem 6.1], one can use Proposition 5.10 to show that, for each $F \in \mathcal{F}$,

$$
\mathcal{U}_{F}:=\overline{\operatorname{span}}\left\{i_{X}(x) i_{B_{P}}\left(1_{s}\right) i_{X}(y)^{*}: \theta(p(x) s)=\theta(p(y) s) \in F\right\}
$$

is a $C^{*}$-subalgebra of $B_{P} \rtimes_{\tau, X} P$. Applying $\Phi_{\delta_{\theta}}$ to both sides of (6.2) gives

$$
\left(B_{P} \rtimes_{\tau, X} P\right)^{\delta_{\theta}}=\overline{\operatorname{span}}\left\{i_{X}(x) i_{B_{P}}\left(1_{s}\right) i_{X}(y)^{*}: \theta(p(x))=\theta(p(y))\right\} ;
$$

since $\mathcal{F}$ is directed under set inclusion (see the proof of [17, Lemma 4.1]), we deduce that

$$
\left(B_{P} \rtimes_{\tau, X} P\right)^{\delta_{\theta}}=\overline{\bigcup_{F \in \mathcal{F}} \mathcal{U}_{F}} .
$$

By [2, Lemma 1.3], to prove that $L^{\Psi} \times \Psi$ is faithful on $\left(B_{P} \rtimes_{\tau, X} P\right)^{\delta_{\theta}}$ it is enough to prove it is faithful on each of the subalgebras $\mathcal{U}_{F}$. We shall accomplish this by inducting on $|F|$.

First suppose $F=\{r\}$ for some $r \in \mathcal{P}$. Let $W_{r}$ be the Hilbert $A-A$ bimodule $\bigoplus_{t \in \theta^{-1}(r)} X_{t}$. We claim that, for each Nica-covariant Toeplitz representation $\psi$ of $X$ on a Hilbert space $\mathcal{K}$, there is a linear map $\psi_{r}$ : $W_{r} \rightarrow B(\mathcal{K})$ which satisfies $\psi_{r}\left(\oplus x_{t}\right)=\sum \psi_{t}\left(x_{t}\right)$, and that $\left(\psi_{r}, \psi_{e}\right)$ is then a Toeplitz representation of $W_{r}$. First observe that if $x, y \in X$ satisfy $p(x) \neq$ $p(y)$ and $\theta(p(x))=\theta(p(y))=r$, then by (8.1) we have $p(x) \vee p(y)=\infty$, and hence $\psi(x)^{*} \psi(y)=0$. Now suppose $\oplus x_{t}$ belongs to the algebraic direct sum $\bigodot_{t \in \theta^{-1}(r)} X_{t}$; such vectors are dense in $W_{r}$. Then

$$
\begin{aligned}
\left\|\sum_{t} \psi_{t}\left(x_{t}\right)\right\|^{2} & =\left\|\sum_{t, t^{\prime}} \psi_{t}\left(x_{t}\right)^{*} \psi_{t^{\prime}}\left(x_{t^{\prime}}\right)\right\|=\left\|\sum_{t} \psi_{t}\left(x_{t}\right)^{*} \psi_{t}\left(x_{t}\right)\right\| \\
& =\left\|\sum_{t} \psi_{e}\left(\left\langle x_{t}, x_{t}\right\rangle_{A}\right)\right\| \leq\left\|\sum_{t}\left\langle x_{t}, x_{t}\right\rangle_{A}\right\| \\
& =\left\|\left\langle\oplus x_{t}, \oplus x_{t}\right\rangle_{A}\right\|=\left\|\oplus x_{t}\right\|^{2},
\end{aligned}
$$

ensuring the existence of $\psi_{r}$. It is routine to check that $\left(\psi_{r}, \psi_{e}\right)$ is a Toeplitz representation of $W_{r}$. Write $\alpha_{r}^{\psi}$ for the endomorphism of $\psi_{e}(A)^{\prime}$ which corresponds to $\left(\psi_{r}, \psi_{e}\right)$ (Proposition 4.1), and write $\rho_{r}^{\psi}$ for the associated representation of $\mathcal{L}\left(W_{r}\right)$ (Lemma 5.5).

Suppose $Z$ is a finite sum $\sum i_{X}\left(x_{k}\right) i_{B_{P}}\left(1_{s_{k}}\right) i_{X}\left(y_{k}\right)^{*}$ such that $\theta\left(p\left(x_{k}\right) s_{k}\right)=$ $\theta\left(p\left(y_{k}\right) s_{k}\right)=r$ for every $k$; to prove $L^{\Psi} \times \Psi$ faithful on $\mathcal{U}_{\{r\}}$ we will show 
that $\left\|L^{\Psi} \times \Psi(Z)\right\|=\|Z\|$. For each $k$, let $\Theta_{x_{k}, y_{k}} \otimes_{A} 1^{s_{k}}$ denote the operator in $\mathcal{L}\left(W_{r}\right)$ which is the image of

$$
\begin{aligned}
& \Theta_{x_{k}, y_{k}} \in \mathcal{K}\left(X_{p\left(y_{k}\right)}, X_{p\left(x_{k}\right)}\right) \mapsto \\
& \Theta_{x_{k}, y_{k}} \otimes_{A} 1^{s_{k}} \in \mathcal{L}\left(X_{p\left(y_{k}\right) s_{k}}, X_{p\left(x_{k}\right) s_{k}}\right) \subset \mathcal{L}\left(W_{r}\right) .
\end{aligned}
$$

Define $T:=\sum \Theta_{x_{k}, y_{k}} \otimes_{A} 1^{s_{k}} \in \mathcal{L}\left(W_{r}\right)$. It is routine to check that

$$
\rho_{r}^{\Psi}(T)=\sum \Psi\left(x_{k}\right) L^{\Psi}\left(1_{s_{k}}\right) \Psi\left(y_{k}\right)^{*}=L^{\Psi} \times \Psi(Z),
$$

and similarly $\rho_{r}^{i}(T)=L^{i} \times i(Z)=\sigma(Z)$. Since $\Psi_{e}$ and $i_{e}$ are faithful representations of $A$, the representations $\rho_{r}^{\Psi}$ and $\rho_{r}^{i}$ are isometric, and thus

$$
\left\|L^{\Psi} \times \Psi(Z)\right\|=\left\|\rho_{r}^{\Psi}(T)\right\|=\|T\|=\left\|\rho_{r}^{i}(T)\right\|=\|\sigma(Z)\|=\|Z\| .
$$

For the inductive step, suppose $F \in \mathcal{F}$ and $L^{\Psi} \times \Psi$ is faithful on $\mathcal{U}_{F^{\prime}}$ whenever $F^{\prime} \in \mathcal{F}$ and $\left|F^{\prime}\right|<|F|$; we aim to prove that $L^{\Psi} \times \Psi$ is faithful on $\mathcal{U}_{F}$. Since $F$ is finite it has a minimal element; that is, there exists $r_{0} \in F$ such that $r_{0}<r_{0} \vee r$ for each $r \in F \backslash\left\{r_{0}\right\}$. As in the proof of [12, Theorem 6.1] we have $L^{\Psi} \times \Psi\left(\mathcal{U}_{\{r\}}\right) P_{r_{0}}=\{0\}$ for each $r \in F \backslash\left\{r_{0}\right\}$, where $P_{r_{0}}$ denotes the orthogonal projection of $F(X) \otimes_{A} \mathcal{H}$ onto $\bigoplus_{t \in \theta^{-1}\left(r_{0}\right)} X_{t} \otimes_{A} \mathcal{H}$.

On the other hand, we have already demonstrated that $L^{\Psi} \times \Psi$ maps $\mathcal{U}_{r_{0}}$ isometrically into the range of $\rho_{r_{0}}^{\Psi}$, and an easy calculation shows that $P_{r_{0}}=\alpha_{r_{0}}^{\Psi}\left(Q_{e}\right)$, where $Q_{e}$ is the orthogonal projection onto $X_{e} \otimes_{A} \mathcal{H}$. Since $a \mapsto \Psi_{e}(a) Q_{e}$ is faithful, by Lemma 5.5(4) the representation $S \in \mathcal{L}\left(W_{r_{0}}\right) \mapsto$ $P_{r_{0}} \rho_{r_{0}}^{\Psi}(S)$ is also faithful. Hence the map $Y \in \mathcal{U}_{r_{0}} \mapsto L^{\Psi} \times \Psi(Y) P_{r_{0}}$ is faithful.

Now suppose $Y \in \mathcal{U}_{F}$ and $L^{\Psi} \times \Psi(Y)=0$. We will show that $Y \in \mathcal{U}_{F \backslash\left\{r_{0}\right\}}$, from which the inductive hypothesis implies that $Y=0$. Let $\left(Y_{n}\right)$ be a sequence in

$$
\operatorname{span}\left\{i_{X}(x) i_{B_{P}}\left(1_{s}\right) i_{X}(y)^{*}: \theta(p(x) s)=\theta(p(y) s) \in F\right\}
$$

which converges in norm to $Y$, and express each $Y_{n}$ as a sum $\sum_{r \in F} Y_{n, r}$, where $Y_{n, r} \in \mathcal{U}_{\{r\}}$. For each $n$,

$$
\left\|L^{\Psi} \times \Psi\left(Y_{n}\right) P_{r_{0}}\right\|=\left\|L^{\Psi} \times \Psi\left(Y_{n, r_{0}}\right) P_{r_{0}}\right\|=\left\|Y_{n, r_{0}}\right\|,
$$

and consequently $Y_{n, r_{0}} \rightarrow 0$. Thus $Y_{n}-Y_{n, r_{0}} \rightarrow Y$, which shows that $Y \in \mathcal{U}_{F \backslash\left\{r_{0}\right\}}$, as claimed.

Corollary 8.2. Suppose $\left(G^{\lambda}, P^{\lambda}\right)$ is a quasi-lattice ordered group with $G^{\lambda}$ amenable for each $\lambda$ belonging to some index set $\Lambda$. If $X$ is a compactlyaligned product system over $P:=* P^{\lambda}$, then the system $\left(B_{P}, P, \tau, X\right)$ is amenable.

Proof. The group $\bigoplus G^{\lambda}$ is amenable, and by [17, Proposition 4.3] the canonical map $\theta: * G^{\lambda} \rightarrow \bigoplus G^{\lambda}$ satisfies (8.1). 


\section{Applications.}

In Section 3, we associated with each twisted semigroup dynamical system $(A, P, \beta, \omega)$ a product system $X=X(A, P, \beta, \omega)$ of essential Hilbert $A$ $A$ bimodules over the opposite semigroup $P^{o}$ (Lemma 3.2), and we showed that the Cuntz-Pimsner algebra $\mathcal{O}_{X}$ is canonically isomorphic to the crossed product $A \rtimes_{\beta, \omega} P$; we also showed that $\mathcal{T}_{X}$ has the structure of a certain "Toeplitz" crossed product $\mathcal{T}\left(A \rtimes_{\beta, \omega} P\right)$ (Proposition 3.4). Suppose now that $\left(G^{o}, P^{o}\right)$ is quasi-lattice ordered; this is equivalent to $(G, P)$ being quasilatticed ordered in its right-invariant partial order $\left(s \leq t \Leftrightarrow t s^{-1} \in P\right)$. Since the left action of $A$ on each fiber $X_{s}$ is by compact operators, $X$ is compactly aligned (Lemma 5.8) and $\mathcal{T}_{\text {cov }}(X)=B_{P} \rtimes_{\tau, X} P$ (Theorem 6.3). Hence we can apply Theorem 7.2 to characterize the faithful representations of $\mathcal{T}_{\text {cov }}(X)$. This is particularly helpful when $\left(G^{o}, P^{o}\right)$ is a total order since $\mathcal{T}_{\text {cov }}(X)=\mathcal{T}_{X}$; more generally, when every $s, t \in P^{o}$ have a common upper bound in $P^{o}$ (i.e., $P s \cap P t \neq \emptyset$ ), the crossed product $A \rtimes_{\beta, \omega} P=\mathcal{O}_{X}$ is a quotient of $\mathcal{T}_{\text {cov }}(X)$ (Theorem 6.3).

We begin by showing that $\mathcal{T}_{\text {cov }}(X)$, too, has a crossed product structure:

Definition 9.1. Suppose $P$ is a subsemigroup of a group $G$ and $\left(G^{o}, P^{o}\right)$ is quasi-lattice ordered. A Nica-Toeplitz covariant representation of $(A, P, \beta, \omega)$ is a Toeplitz covariant representation $(\pi, V)$ such that

$$
V_{s}^{*} V_{s} V_{t}^{*} V_{t}= \begin{cases}V_{s \vee t}^{*} V_{s \vee t} & \text { if } s \vee t<\infty \\ 0 & \text { otherwise, }\end{cases}
$$

where $s \vee t$ denotes the least upper bound of $s$ and $t$ in the right-invariant partial order on $(G, P)$.

The following Proposition establishes the existence of a $C^{*}$-algebra which is universal for such pairs $(\pi, V)$, as in Definition 3.1. We call this algebra the Nica-Toeplitz crossed product of $(A, P, \beta, \omega)$, and denote it $\mathcal{T}_{\text {cov }}\left(A \rtimes_{\beta, \omega} P\right)$. Let $i_{X}: X \rightarrow \mathcal{T}_{\text {cov }}(X)$ be universal for Nica-covariant Toeplitz representations of $X$. Lemma 3.3 is easily adapted to this setting, and allows us to define $i_{P}: P \rightarrow M \mathcal{T}_{\text {cov }}(X)$ by $i_{P}(s)=\lim i_{X}\left(s, \beta_{s}\left(a_{i}\right)\right)^{*}$; here $\left(a_{i}\right)$ is an approximate identity for $A$, and the convergence is strict. We also define $i_{A}: A \rightarrow \mathcal{T}_{\text {cov }}(X)$ by $i_{A}(a):=i_{X}(e, a)$.

Proposition 9.2. $\left(\mathcal{T}_{\text {cov }}(X), i_{A}, i_{P}\right)$ is a Nica-Toeplitz crossed product for $(A, P, \beta, \omega)$.

Proof. As in the proof of Proposition 3.4, $i_{A}$ is nondegenerate. We verify the obvious analogues of Conditions (a), (b), and (c) in Definition 3.1. For (a), let $\sigma$ be a nondegenerate representation of $\mathcal{T}_{\text {cov }}(X)$ on a Hilbert space $\mathcal{H}$, let $\pi:=\sigma \circ i_{A}$, and let $V:=\bar{\sigma} \circ i_{P}$; we must show that $(\pi, V)$ is a NicaToeplitz covariant representation of $(A, P, \beta, \omega)$. Exactly as in the proof of 
Proposition 3.4, $(\pi, V)$ is a Toeplitz covariant representation of $(A, P, \beta, \omega)$, so we need to establish (9.1). Fix $s \in P$. For any $a \in A$ and $h \in \mathcal{H}$ we have

$$
\begin{aligned}
V_{s}^{*} \pi(a) h & =\sigma\left(i_{P}(s)^{*} i_{A}(a)\right) h=\sigma\left(\lim i_{X}\left(s, \beta_{s}\left(a_{i}\right)\right) i_{X}(e, a)\right) h \\
& =\sigma\left(\lim i_{X}\left(s, \beta_{s}\left(a_{i}\right) a\right)\right) h=\sigma \circ i_{X}\left(s, \overline{\beta_{s}}(1) a\right) h,
\end{aligned}
$$

and since $\pi$ is nondegenerate this shows that

$$
V_{s}^{*} V_{s} \mathcal{H}=\overline{\operatorname{span}}\left\{\sigma \circ i_{X}(\xi) h: \xi \in X_{s}, h \in \mathcal{H}\right\}=\alpha_{s}^{\sigma \circ i_{X}}(1) .
$$

Since $X$ is compactly aligned, $\sigma \circ i_{X}$ is Nica covariant (Theorem 6.3 and Proposition 5.9), and (9.1) follows.

For Condition (b), let $(\pi, V)$ be any Nica-Toeplitz covariant representation on $\mathcal{H}$. As in the proof of Proposition 3.4, $\psi(s, x):=V_{s}^{*} \pi(x)$ defines a nondegenerate Toeplitz covariant representation $\psi: X \rightarrow B(\mathcal{H})$. To see that it is Nica-covariant, let $s \in P$, and note that for any $a \in A$ we have

$$
\begin{aligned}
\psi\left(s, \overline{\beta_{s}}(1) a\right) & =\lim \psi\left(s, \beta_{s}\left(a_{i}\right) a\right)=\lim V_{s}^{*} \pi\left(\beta_{s}\left(a_{i}\right) a\right) \\
& =\lim V_{s}^{*} V_{s} \pi\left(a_{i}\right) V_{s}^{*} \pi(a)=V_{s}^{*} \pi(a) .
\end{aligned}
$$

Since $\pi$ is nondegenerate, this implies that $\alpha_{s}^{\psi}(1)=V_{s}^{*} V_{s}$, and hence $\psi$ is Nica covariant by (9.1). Defining $\pi \times V:=\psi_{*}: \mathcal{T}_{\text {cov }}(X) \rightarrow B(\mathcal{H})$ gives the desired representation satisfying $(\pi \times V) \circ i_{A}=\pi$ and $\overline{\pi \times V} \circ i_{P}=V$. Condition (c) is satisfied since $i_{A}(a) i_{P}(s)=i_{X}\left(s, \overline{\beta_{s}}(1) a^{*}\right)^{*}$, and elements of this form generate $\mathcal{T}_{\text {cov }}(X)$.

Let $\left(G_{i}, P_{i}\right)$ be a collection of abelian lattice-ordered groups. Since $\left(G_{i}, P_{i}\right)$ is quasi-lattice ordered in both its left and its right-invariant partial order, so is the free product $*\left(G_{i}, P_{i}\right)$.

Theorem 9.3. Suppose $(G, P)=*\left(G_{i}, P_{i}\right)$ is a free product of abelian lattice-ordered groups and $(\pi, V)$ is a Nica-Toeplitz covariant representation of the twisted semigroup dynamical system $(A, P, \beta, \omega)$ on a Hilbert space $\mathcal{H}$. Then the integrated form $\pi \times V$ is a faithful representation of $\mathcal{T}_{\text {cov }}\left(A \rtimes_{\beta, \omega} P\right)$ if and only if

$$
\text { for every } n \geq 1 \text { and } s_{1}, \ldots, s_{n} \in P \backslash\{e\},
$$$$
\pi \text { acts faithfully on the range of } \prod_{k=1}^{n}\left(1-V_{s_{k}}^{*} V_{s_{k}}\right) \text {. }
$$

Proof. Let $\theta$ be the canonical homomorphism of $*\left(G_{i}, P_{i}\right)$ onto $\bigoplus\left(G_{i}, P_{i}\right)$. By [17, Proposition 4.3], $\theta$ satisfies the hypotheses of Theorem 8.1; since $X=X(A, P, \beta, \omega)$ is compactly aligned, the system $\left(B_{P}, P, \tau, X\right)$ is therefore amenable. Identifying $\mathcal{T}_{\text {cov }}\left(A \rtimes_{\beta, \omega} P\right)$ with $\mathcal{T}_{\text {cov }}(X)$ as in the previous Proposition and defining $\psi(s, x):=V_{s}^{*} \pi(x)$, the initial projection $V_{s}^{*} V_{s}$ is precisely $\alpha_{s}^{\psi}(1)$, and the result follows from Theorem 7.2. 
Nica covariance is automatic when $(G, P)$ is totally ordered:

Corollary 9.4. Suppose $(G, P)$ is a totally ordered abelian group and $(\pi, V)$ is a Toeplitz covariant representation of $(A, P, \beta, \omega)$ on a Hilbert space $\mathcal{H}$. Then the integrated form $\pi \times V$ is a faithful representation of $\mathcal{T}\left(A \rtimes_{\beta, \omega} P\right)$ if and only if $\pi$ acts faithfully on $\left(V_{s}^{*} \mathcal{H}\right)^{\perp}$ for every $s \in P \backslash\{e\}$.

Corollary 9.5. Suppose $\beta$ is an extendible endomorphism of $A$. If $(\pi, V)$ is a Toeplitz representation of $(A, \mathbb{N}, \beta)$, then $\pi \times V$ is a faithful representation of $\mathcal{T}\left(A \rtimes_{\beta} \mathbb{N}\right)$ if and only if $\pi$ acts faithfully on $\left(V^{*} \mathcal{H}\right)^{\perp}$.

Bicovariance. Suppose $(G, P)$ is a quasi-lattice ordered group. Following [17], in [12] it was shown that $B_{P} \rtimes_{\tau, \omega} P$ is universal for isometric $\omega$-representations of $P$ which are Nica covariant; that is, which satisfy

$$
V_{s} V_{s}^{*} V_{t} V_{t}^{*}= \begin{cases}V_{s \vee t} V_{s \vee t}^{*} & \text { if } s \vee t<\infty \\ 0 & \text { otherwise. }\end{cases}
$$

Assuming that $\left(G^{o}, P^{o}\right)$ is also quasi-lattice ordered, we now show that the Nica-Toeplitz crossed product $\mathcal{T}_{\text {cov }}\left(B_{P} \rtimes_{\tau, \omega} P\right)$ is universal for partial isometric $\omega$-representations of $P$ which are bicovariant in that they satisfy both (9.2) and (9.1). Note that bicovariance is automatic when $(G, P)$ is a totally ordered abelian group.

Proposition 9.6. $i_{P}: P \rightarrow \mathcal{T}_{\operatorname{cov}}\left(B_{P} \rtimes_{\tau, \omega} P\right)$ is a bicovariant partial isometric $\omega$-representation of $P$ whose range generates $\mathcal{T}_{\text {cov }}\left(B_{P} \rtimes_{\tau, \omega} P\right)$ as a $C^{*}$-algebra. Moreover, for every bicovariant partial isometric $\omega$-representation $V$, there is a representation $V_{*}$ of $\mathcal{T}_{\text {cov }}\left(B_{P} \rtimes_{\tau, \omega} P\right)$ such that $V_{*} \circ i_{P}=V$.

Proof. Let $\sigma$ be a faithful nondegenerate representation of $\mathcal{T}_{\text {cov }}\left(B_{P} \rtimes_{\tau, \omega} P\right)$. Then $V:=\bar{\sigma} \circ i_{P}$ is a partial isometric $\omega$-representation of $P$ which satisfies (9.1), and applying $\bar{\sigma}^{-1}$ we see that $i_{P}$ is as well. Since $i_{P}(s) i_{P}(s)^{*}=i_{B_{P}}\left(1_{s}\right)$ for every $s \in P, i_{P}$ also satisfies (9.2), and is hence bicovariant. Since $\left\{1_{s}: s \in P\right\}$ generates $B_{P}$ linearly and $\left\{i_{B_{P}}(a) i_{P}(t): a \in B_{P}, t \in P\right\}$ generates $\mathcal{T}_{\text {cov }}\left(B_{P} \rtimes_{\tau, \omega} P\right)$ as a $C^{*}$-algebra, elements of the form $i_{B_{P}}\left(1_{s}\right) i_{P}(t)=$ $i_{P}(s) i_{P}(s)^{*} i_{P}(t)$ are also generating. If $V$ is any bicovariant partial isometric $\omega$-representation of $P$, then by $[\mathbf{1 7}$, Proposition 1.3] there is a representation $\pi_{V}$ of $B_{P}$ such that $\pi_{V}\left(1_{s}\right)=V_{s} V_{s}^{*}$ for every $s \in P$. For any $s, t \in P$ the product $V_{t} V_{s}=\omega(t, s) V_{t s}$ is a partial isometry; hence by [14, Lemma 2] the projections $V_{s} V_{s}^{*}$ and $V_{t}^{*} V_{t}$ commute, and we deduce that $\pi_{V}(a) V_{t}^{*} V_{t}=V_{t}^{*} V_{t} \pi_{V}(a)$ for every $a \in B_{P}$ and $t \in P$. Further,

$$
\begin{aligned}
\pi_{V}\left(\tau_{s}\left(1_{t}\right)\right) & =\pi_{V}\left(1_{s t}\right)=V_{s t} V_{s t}^{*} \\
& =\left(\overline{\omega(s, t)} V_{s} V_{t}\right)\left(\overline{\omega(s, t)} V_{s} V_{t}\right)^{*}=V_{s} V_{t} V_{t}^{*} V_{s}^{*}=V_{s} \pi_{V}\left(1_{t}\right) V_{s}^{*},
\end{aligned}
$$

so $\pi_{V}\left(\tau_{s}(a)\right)=V_{s} \pi_{V}(a) V_{s}^{*}$ for every $s \in P$ and $a \in B_{P}$. Thus $\left(\pi_{V}, V\right)$ is a Nica-Toeplitz covariant representation of $\left(B_{P}, P, \tau, \omega\right)$. The representation $V_{*}:=\pi_{V} \times V$ satisfies $V_{*} \circ i_{P}=V$. 
We say that a bicovariant partial isometric $\omega$-representation $V$ is universal if, for every bicovariant partial isometric $\omega$-representation $W$, there is a homomorphism of $C^{*}\left\{V_{s}: s \in P\right\}$ which maps $V_{s}$ to $W_{s}$ for each $s \in P$.

Theorem 9.7. Suppose $(G, P)=*\left(G_{i}, P_{i}\right)$ is a free product of abelian lattice-ordered groups and $V$ is a bicovariant partial isometric $\omega$-representation of $P$. Then $V$ is universal if and only if

$$
\prod_{l=1}^{m}\left(V_{r} V_{r}^{*}-V_{r t_{l}} V_{r t_{l}}^{*}\right) \prod_{k=1}^{n}\left(1-V_{s_{k}}^{*} V_{s_{k}}\right) \neq 0
$$

whenever $r \in P, m, n \geq 1$, and $s_{1}, \ldots, s_{n}, t_{1}, \ldots, t_{m} \in P \backslash\{e\}$.

Proof. $V$ is universal if and only if the representation $V_{*}=\pi_{V} \times V$ of $\mathcal{T}_{\text {cov }}\left(B_{P} \rtimes_{\tau, \omega} P\right)$ is faithful. By Theorem 9.3, this occurs if and only if $\pi_{V}$ acts faithfully on the range of $\prod_{k=1}^{n}\left(1-V_{s_{k}}^{*} V_{s_{k}}\right)$ whenever $s_{1}, \ldots, s_{n} \in P \backslash\{e\}$, and the result follows from [17, Proposition 1.3].

Let $\mathbb{F}_{\infty}$ be the free group on infinitely many generators $z_{1}, z_{2}, \ldots$, and let $\mathbb{F}_{\infty}^{+}$be the subsemigroup (with identity) generated by the $z_{i}$; the pair $\left(\mathbb{F}_{\infty}, \mathbb{F}_{\infty}^{+}\right)$is quasi-lattice ordered. In [17], Laca and Raeburn realized the Cuntz algebra $\mathcal{O}_{\infty}$ as the universal $C^{*}$-algebra for covariant isometric representations of $\mathbb{F}_{\infty}^{+}$, and used their characterization of the faithful representations of $B_{P} \rtimes_{\tau} P$ to derive Cuntz's simplicity result. We finish by showing that the universal $C^{*}$-algebra for bicovariant partial isometric representations of $\mathbb{F}_{\infty}^{+}$is reminiscent of $\mathcal{O}_{\infty}$, and we derive a Cuntz-Krieger-type uniqueness theorem.

First some notation. For a multi-index $\mu=\left(\mu_{1}, \ldots, \mu_{n}\right)$ we write $z_{\mu}:=$ $z_{\mu_{1}} \cdots z_{\mu_{n}}$, and we identify $\mathbb{F}_{\infty}^{+}$with the set of multi-indices under concatenation via $z_{\mu} \leftrightarrow \mu$.

Proposition 9.8. Suppose $S$ is a partial isometric representation of $\mathbb{F}_{\infty}^{+}$in a $C^{*}$-algebra $B$; that is, $S$ is a semigroup homomorphism and each $S_{\mu}$ is a partial isometry. Then $C^{*}\left\{S_{\mu}: \mu \in \mathbb{F}_{\infty}^{+}\right\}$is generated by $\left\{S_{n}: n \in \mathbb{N}\right\}$, and $S$ is bicovariant if and only if

(a) the range projections $s_{k} s_{k}^{*}$ for $k \in \mathbb{N}$ are pairwise orthogonal, and

(b) the initial projections $s_{k}^{*} s_{k}$ for $k \in \mathbb{N}$ are pairwise orthogonal.

Proof. The first statement is obvious. In the left-invariant partial order on $\mathbb{F}_{\infty}$, two elements $\mu, \nu \in \mathbb{F}_{\infty}^{+}$have a common upper bound if and only if one is an initial word of the other, and then the least upper bound is the longer of the two words. We will show that (a) holds if and only if

$$
S_{\mu} S_{\mu}^{*} S_{\nu} S_{\nu}^{*}= \begin{cases}S_{\mu} S_{\mu}^{*} & \text { if } \nu^{-1} \mu \in \mathbb{F}_{\infty}^{+}, \\ S_{\nu} S_{\nu}^{*} & \text { if } \mu^{-1} \nu \in \mathbb{F}_{\infty}^{+} \\ 0 & \text { otherwise }\end{cases}
$$


of course a similar statement holds for (b) using the right-invariant partial order, and together these prove the Proposition.

To begin with, (9.3) implies (a) since distict generators of $\mathbb{F}_{\infty}^{+}$are not comparable. For the converse, first suppose $\nu^{-1} \mu \in \mathbb{F}_{\infty}^{+}$; since $S_{\nu}$ is a partial isometry, we then have

$$
S_{\mu} S_{\mu}^{*} S_{\nu} S_{\nu}^{*}=S_{\mu} S_{\nu^{-1} \mu}^{*} S_{\nu}^{*} S_{\nu} S_{\nu}^{*}=S_{\mu} S_{\nu^{-1} \mu}^{*} S_{\nu}^{*}=S_{\mu} S_{\mu}^{*}
$$

The case $\mu^{-1} \nu \in \mathbb{F}_{\infty}^{+}$is similar. Finally, suppose $\mu$ and $\nu$ are not comparable. Then there exists $\sigma, \mu^{\prime}, \nu^{\prime} \in \mathbb{F}_{\infty}^{+}$such that $\mu=\sigma \mu^{\prime}, \nu=\sigma \nu^{\prime}$, and $\mu_{1}^{\prime} \neq \nu_{1}^{\prime}$. Condition (a) implies that $S_{\mu^{\prime}}^{*} S_{\nu^{\prime}}=0$, and by [14, Lemma 2] the range projection of $S_{\nu^{\prime}}$ commutes with the initial projection of $S_{\sigma}$, so

$$
S_{\mu}^{*} S_{\nu}=S_{\mu^{\prime}}^{*} S_{\sigma}^{*} S_{\sigma} S_{\nu^{\prime}}=S_{\mu^{\prime}}^{*} S_{\sigma}^{*} S_{\sigma} S_{\nu^{\prime}} S_{\nu^{\prime}}^{*} S_{\nu^{\prime}}=S_{\mu^{\prime}}^{*} S_{\nu^{\prime}} S_{\nu^{\prime}}^{*} S_{\sigma}^{*} S_{\sigma} S_{\nu^{\prime}}=0 .
$$

Theorem 9.9. A bicovariant partial isometric representation $S$ of $\mathbb{F}_{\infty}^{+}$is universal if and only if each $S_{\mu}$ is nonzero.

Proof. Suppose each $S_{\mu}$ is nonzero. To see that $S$ is universal, we apply Theorem 9.7. If $\nu \in \mathbb{F}_{\infty}^{+}, m, n \geq 1$, and $\sigma_{1}, \ldots, \sigma_{m}, \tau_{1}, \ldots, \tau_{n} \in \mathbb{F}_{\infty}^{+} \backslash\{e\}$, then we can choose $i, j \in \mathbb{N}$ such that none of the multi-indices $\sigma_{l}$ begins with $i$, and none of the multi-indices $\tau_{k}$ ends with $j$. Then

$$
\prod_{l=1}^{m}\left(S_{\nu} S_{\nu}^{*}-S_{\nu \sigma_{l}} S_{\nu \sigma_{l}}^{*}\right) \prod_{k=1}^{n}\left(1-S_{\tau_{k}}^{*} S_{\tau_{k}}\right) \geq S_{\nu} S_{i} S_{i}^{*} S_{\nu}^{*} S_{j}^{*} S_{j}=S_{j}^{*} S_{j \nu i} S_{j \nu i}^{*} S_{j}
$$

is nonzero since $S_{j}\left(S_{j}^{*} S_{j \nu i} S_{j \nu i}^{*} S_{j}\right) S_{j}^{*}=S_{j \nu i} S_{j \nu i}^{*} \neq 0$. Hence $S$ is universal.

Now define $T: \mathbb{F}_{\infty}^{+} \rightarrow B\left(\ell^{2}\left(\mathbb{F}_{\infty}^{+}\right) \otimes \ell^{2}\left(\mathbb{F}_{\infty}^{+}\right)\right)$by

$$
T_{\mu}\left(\delta_{\sigma} \otimes \delta_{\nu}\right)= \begin{cases}\delta_{\sigma} \otimes \delta_{\mu \nu} & \text { if } \sigma \text { ends in } \mu \nu \\ 0 & \text { otherwise. }\end{cases}
$$

Then $T$ is a bicovariant partial isometric representation of $\mathbb{F}_{\infty}^{+}$in which each $T_{\mu}$ is nonzero. If $S$ is universal, then $S_{\mu} \mapsto T_{\mu}$ extends to a homomorphism of $C^{*}\left\{S_{\mu}\right\}$, and hence each $S_{\mu}$ must be nonzero.

\section{References}

[1] S. Adji, Ph.D. Thesis, Univ. of Newcastle, 1995.

[2] S. Adji, M. Laca, M. Nilsen and I. Raeburn, Crossed products by semigroups of endomorphisms and the Toeplitz algebras of ordered groups, Proc. Amer. Math. Soc., 122 (1994), 1133-1141, MR 95b:46094, Zbl 0818.46071.

[3] W. Arveson, Continuous analogues of Fock space, Memoirs Amer. Math. Soc., 80(409) (1989), MR 90f:47061, Zbl 0697.46035. 
[4] S. Boyd, N. Keswani and I. Raeburn, Faithful representations of crossed products by endomorphisms, Proc. Amer. Math. Soc., 118 (1993), 427-436, MR 93g:46066, Zbl 0785.46051.

[5] J. Cuntz, Simple $C^{*}$-algebras generated by isometries, Comm. Math. Phys., 57 (1977), 173-185, MR 57 \#7189, Zbl 0399.46045.

[6] _ The internal structure of simple $C^{*}$-algebras, Proceedings of Symposia in Pure Mathematics, 38(1) (1982), 85-115, MR 84h:46072, Zbl 0502.46039.

[7] H.T. Dinh, Discrete product systems and their $C^{*}$-algebras, J. Funct. Anal., 102 (1991), 1-34, MR 93d:46097, Zbl 0745.46057.

[8] _ On generalized Cuntz $C^{*}$-algebras, J. Operator Theory, 30 (1993), 123-135, MR 95m:46112, Zbl 0837.46048.

[9] S. Doplicher, C. Pinzari and R. Zuccante, The $C^{*}$-algebra of a Hilbert bimodule, Boll. Unione Mat. Ital. Sez. B Artic. Ric. Mat., 8(1) (1998), 263-281, MR 99i:46040, Zbl 0916.46053.

[10] N.J. Fowler, Compactly-aligned discrete product systems, and generalizations of $\mathcal{O}_{\infty}$, International J. Math., 10(6) (1999), 721-738, CMP 1715181.

[11] N.J. Fowler, P.S. Muhly and I. Raeburn, Representations of Cuntz-Pimsner algebras, to appear in Indiana Univ. Math. J.

[12] N.J. Fowler and I. Raeburn, Discrete product systems and twisted crossed products by semigroups, J. Funct. Anal., 155 (1998), 171-204, MR 99k:46118, Zbl 0914.46054.

[13] _ The Toeplitz algebra of a Hilbert bimodule, Indiana Univ. Math. J., 48(1) (1999), 155-181, MR 2001b:46093, Zbl 0938.47052.

[14] P.R. Halmos and L.J. Wallen, Powers of partial isometries, Indiana Univ. Math. J., 19 (1970), 657-663, MR 40 \#4801, Zbl 0202.42502.

[15] R. Hancock and I. Raeburn, The $C^{*}$-algebras of some inverse semigroups, Bull. Austral. Math. Soc., 42 (1990), 335-348, MR 91i:46061, Zbl 0716.46052.

[16] T. Kajiwara, C. Pinzari and Y. Watatani, Ideal structure and simplicity of the $C^{*}$-algebras generated by Hilbert bimodules, J. Funct. Anal., 159 (1998), 295-322, MR 2000a:46094, Zbl 0942.46035.

[17] M. Laca and I. Raeburn, Semigroup crossed products and the Toeplitz algebras of nonabelian groups, J. Funct. Anal., 139 (1996), 415-440, MR 97h:46109, Zbl 0887.46040.

[18] P.S. Muhly and B. Solel, On the simplicity of some Cuntz-Pimsner algebras, Math. Scand., 83 (1998), 53-73, MR 99m:46140, Zbl 0940.46034.

[19] - Tensor algebras over $C^{*}$-correspondences (representations, dilations, and $C^{*}$ envelopes), J. Funct. Anal., 158 (1998), 389-457, MR 99j:46066, Zbl 0912.46070.

[20] A. Nica, $C^{*}$-algebras generated by isometries and Wiener-Hopf operators, J. Operator Theory, 27 (1992), 17-52, MR 94m:46094, Zbl 0809.46058.

[21] W.L. Paschke, The crossed product by an endomorphism, Proc. Amer. Math. Soc., 80 (1980), 113-118, MR 81m:46081, Zbl 0435.46045.

[22] M.V. Pimsner, A class of $C^{*}$-algebras generalizing both Cuntz-Krieger algebras and crossed products by $\mathbb{Z}$, Fields Institute Communications, 12 (1997), 189-212, MR 97k:46069, Zbl 0871.46028.

[23] I. Raeburn and D.P. Williams, Morita equivalence and continuous-trace $C^{*}$-algebras, Math. Surveys and Monographs, 60 (1998), Amer. Math. Soc., Providence, MR 2000c:46108, Zbl 0922.46050. 
[24] P.J. Stacey, Crossed products of $C^{*}$-algebras by endomorphisms, J. Austral. Math. Soc. (Series A), 54 (1993), 204-212, MR 94a:46077, Zbl 0785.46053.

Received April 25, 1999. The author thanks the University of Victoria, Canada, for its hospitality while this research was being completed.

3316 179Th Avenue NE

REDMOND WA 98052

E-mail address: duvalfowler@msn.com 